\title{
BACKWARD UNIQUENESS FOR PARABOLIC OPERATORS WITH VARIABLE COEFFICIENTS IN A HALF SPACE
}

\author{
JIE WU， LIQUN ZHANG*
}

\begin{abstract}
It is shown that a function $u$ satisfying $\left|\partial_{t} u+\sum_{i, j} \partial_{i}\left(a^{i j} \partial_{j} u\right)\right| \leq N(|u|+$ $|\nabla u|),|u(x, t)| \leq N e^{N|x|^{2}}$ in $\mathbb{R}_{+}^{n} \times[0, T]$ and $u(x, 0)=0$ in $\mathbb{R}_{+}^{n}$ under certain conditions on $\left\{a^{i j}\right\}$ must vanish identically in $\mathbb{R}_{+}^{n} \times[0, T]$. The main point of the result is that the conditions imposed on $\left\{a^{i j}\right\}$ are of this type: $\left\{a^{i j}\right\}$ are Lipschitz and $\left|\nabla_{x} a^{i j}(x, t)\right| \leq \frac{E}{|x|}$, where $E$ is less than a given number, and the conditions are optimal in some sense.
\end{abstract}

Keywords: Carleman estimates; Backward uniqueness; Landis and Oleinik; Parabolic operator; Variable Coefficient.

Mathematics Subject Classification: 35K10; 35A02; 35R45.

\section{INTRODUCTION}

Let $U$ be a domain in $\mathbb{R}^{n}$ and $P$ be a backward parabolic operator on $U \times[0, T]$,

$$
P=\partial_{t}+\sum_{i, j} \partial_{i}\left(a^{i j} \partial_{j}\right)=\partial_{t}+\nabla \cdot(A \nabla),
$$

where $A(x, t)=\left(a^{i j}(x, t)\right)_{i, j=1}^{n}$ is a real symmetric matrix such that for some $\Lambda \geq \lambda>0$,

$$
\lambda|\xi|^{2} \leq \sum_{i, j} a^{i j}(x, t) \xi_{i} \xi_{j} \leq \Lambda|\xi|^{2}, \forall \xi \in \mathbb{R}^{n}
$$

Consider a function $u$ which satisfies

$$
\begin{cases}|P u| \leq N(|u|+|\nabla u|) & \text { in } U \times[0, T], \\ |u(x, t)| \leq N e^{N|x|^{2}} & \text { in } U \times[0, T], \\ u(x, 0)=0 & \text { in } U,\end{cases}
$$

where $N$ is a given positive constant.

The backward uniqueness $(\mathrm{BU})$ problem is: does $u$ vanish identically in $U \times[0, T]$ ? If so, we say that $U$ is a $\mathrm{BU}$ domain for the operator $P$.

We should point out that there is no boundary condition about $u$ on the boundary of the domain $U$. The BU problem is recently discussed by L. Escauriaza, G. Seregin and V. Šverák in [7]. It appeared in many problems, for example, in the control theory for PDEs and the regularity theory of parabolic equations. Especially, it plays an important role in the regularity theory of the Navier-Stokes equations, see [9].

Date: June, 2013.

*The research is partially supported by the Chinese NSF under grant 10325104, the innovation program at CAS and National Basic Research Program of China under grant 2011CB808002. 
When $P$ is the backward heat operator, there are many results already on various domains, such as, on the exterior of a ball $\mathbb{R}^{n} \backslash B_{R}$ [7] and a half space $\mathbb{R}_{+}^{n}=\{x \in$ $\left.\mathbb{R}^{n} \mid x_{n}>0\right\}$ [8, 9] by L. Escauriaza, G. Seregin and V. Šverák, and on some cones by Lu Li and V. Šverák [16]. Those are all proved to be BU domains for the backward heat operator. On the other hand, any bounded domain is not BU domain, see [2, 3].

When $P$ is in general, variable coefficients, there are few results have been proved, while some related results have already been obtained. In particular, L. Escauriaza and F. J. Fernández proved a unique continuation property when $\left\{a^{i j}\right\}$ are Lipschitz in [10]. Then it implies immediately that if $U \subset V$ and $U$ is a BU domain, so is $V$. Recently, $\mathrm{Tu} \mathrm{A}$. Nguyen in [18] proved a conjecture of E. M. Landis and O. A. Oleinik [17] which implies that $\mathbb{R}^{n}$ and $\mathbb{R}_{+}^{n}$ are BU domains under the conditions that $\left|\nabla_{x} a^{i j}(x, t)\right|$ and $\left|\partial_{t} a^{i j}(x, t)\right|$ are bounded and the decay at infinity conditions that

$$
\left|\nabla_{x} a^{i j}(x, t)\right| \leq M\langle x\rangle^{-1-\varepsilon},\left|a^{i j}(x, t)-a^{i j}(x, s)\right| \leq M\langle x\rangle^{-1}|t-s|^{1 / 2},
$$

where $\langle x\rangle=\sqrt{1+|x|^{2}}$ and $\varepsilon>0$.

This paper can be regarded as a continuation of the above results. Since $\mathbb{R}^{n} \backslash B(R) \subset \mathbb{R}^{n}$ and $\mathbb{R}_{+}^{n}$ can be treated as a subset of $\mathbb{R}^{n} \backslash B(R)$, by the unique continuation property, we could only consider the case of $\mathbb{R}_{+}^{n}$. Also general simply connected domains may be mapped onto $\mathbb{R}_{+}^{n}$. Then we focus on operator $P$ with variable coefficients on the domain $\mathbb{R}_{+}^{n}$. Our main result is the following.

Theorem 1.1. Suppose $\left\{a^{i j}\right\}$ satisfy (1), and for some constants $E, M, N>0$,

$$
\left|\nabla_{x} a^{i j}(x, t)\right|+\left|\partial_{t} a^{i j}(x, t)\right| \leq M, \forall(x, t) \in \mathbb{R}_{+}^{n} \times[0, T]
$$

and

$$
\left|\nabla_{x} a^{i j}(x, t)\right| \leq \frac{E}{|x|}, \forall(x, t) \in \mathbb{R}_{+}^{n} \times[0, T]
$$

Assume that $u$ satisfies

$$
\begin{cases}|P u| \leq N(|u|+|\nabla u|) & \text { in } \mathbb{R}_{+}^{n} \times[0, T] \\ |u(x, t)| \leq N e^{N|x|^{2}} & \text { in } \mathbb{R}_{+}^{n} \times[0, T], \\ u(x, 0)=0 & \text { in } \mathbb{R}_{+}^{n} .\end{cases}
$$

Then there exists a constant $E_{0}=E_{0}(n, \Lambda, \lambda)$, such that when $E<E_{0}, u(x, t) \equiv 0$ in $\mathbb{R}_{+}^{n} \times[0, T]$.

We remark that our assumptions are optimal in some sense. From the counterexamples constructed by A. Plis [20], K. Miller [21] and N. Mandache [22], we can see that to ensure $\mathrm{BU}$, certain regularity of the coefficients should be required. Moreover, Tu A. Nguyen proved in [18] that the regularity conditions (3) and the decay at infinity conditions (2) will be sufficient to ensure BU. However, are the decay at infinity conditions necessary? Or, are conditions (3) alone enough to guarantee BU? Here, we show that conditions (3) are not enough and the decay at infinity conditions (41) in Theorem $1.1,\left|\nabla_{x} a^{i j}(x, t)\right| \leq \frac{E}{|x|}$, where $E$ is small, are optimal in some sense.

First, we copy the examples given by N. Mandache in [22].

Proposition 1.2. There exist smooth functions $u, b_{11}, b_{12}, b_{22}$ and continuous functions $d_{1}, d_{2}$ defined on $\mathbb{R}^{3} \ni(s, x, y)$, with the following properties: 
i) $u$ is the solution of the equation

$$
\partial_{s}^{2} u+\partial_{x}\left(\left(b_{11}+d_{1}\right) \partial_{x} u\right)+\partial_{y}\left(b_{12} \partial_{x} u\right)+\partial_{x}\left(b_{12} \partial_{y} u\right)+\partial_{y}\left(\left(b_{22}+d_{2}\right) \partial_{y} u\right)=0 .
$$

ii) There is a $T>0$ such that supp $u=(-\infty, T] \times \mathbb{R}^{2}$.

iii) $u, b_{i j}$ and $d_{i}$ are periodic in $x$ and in $y$ with period $2 \pi$.

iv) $d_{1}$ and $d_{2}$ do not depend on $x$ and $y$ and are Hölder continuous of order $\alpha$ for all $\alpha<1$.

v) $\frac{1}{2}<\left(\begin{array}{cc}d_{1}+b_{11} & b_{12} \\ b_{12} & d_{2}+b_{22}\end{array}\right)<2$ on $\mathbb{R}^{3}$.

Furthermore, there are also functions as above, satisfying conditions i)-v) except that (6) is replaced with the parabolic equation:

$$
\partial_{s} u=\partial_{x}\left(\left(b_{11}+d_{1}\right) \partial_{x} u\right)+\partial_{y}\left(b_{12} \partial_{x} u\right)+\partial_{x}\left(b_{12} \partial_{y} u\right)+\partial_{y}\left(\left(b_{22}+d_{2}\right) \partial_{y} u\right) .
$$

The solution of (17) implies that the Hölder regularity in the time variable is not enough for BU. Hence it is reasonable for us to assume that $\left|\partial_{t} a^{i j}(x, t)\right|$ are bounded in Theorem 1.1.

Next we consider the requirement of the regularity in the space variable. Assume that $u$ is the solution of ([6) . We denote that

$$
\begin{aligned}
v(t, s, x, y) & =u(T+s+t, x, y) \\
\bar{b}_{i j}(t, s, x, y) & =b_{i j}(T+s+t, x, y) \\
\bar{d}_{i}(t, s) & =d_{i}(T+s+t) .
\end{aligned}
$$

in

$$
[-1,0] \times \mathbb{R}_{+}^{3}=\{(t, s, x, y) \mid t \in[-1,0], s>0, x \in \mathbb{R}, y \in \mathbb{R}\}
$$

Then

$$
\partial_{t} v-\left[\partial_{s}^{2} v+\partial_{x}\left(\left(\overline{b_{11}}+\overline{d_{1}}\right) \partial_{x} v\right)+\partial_{y}\left(\overline{b_{12}} \partial_{x} v\right)+\partial_{x}\left(-\overline{b_{12}} \partial_{y} v\right)+\partial_{y}\left(\left(b_{22}^{-}+\bar{d}_{2}\right) \partial_{y} v\right)\right]-\partial_{s} v=0 .
$$

By $i i)$ of Proposition [1.2, $v(0, s, x, y)=0$ and $v$ is nonzero in $[-1,0] \times \mathbb{R}_{+}^{3}$, thus BU fails. It shows that the Hölder regularity in the space variable is not enough for BU, hence it is also reasonable for us to assume that $\left|\nabla_{x} a^{i j}(x, t)\right|$ are bounded in Theorem 1.1.

Now we consider the decay at infinity conditions. We could construct an example as follows. Consider a cone $\mathcal{C}_{\theta_{0}}$ with opening angle $\theta_{0}$ and the system

$$
\begin{cases}\partial_{t} u+\Delta u=0 & \text { in } \mathcal{C}_{\theta_{0}} \times[0, T], \\ |u(x, t)| \leq N & \text { in } \mathcal{C}_{\theta_{0}} \times[0, T], \\ u(x, 0)=0 & \text { in } \mathcal{C}_{\theta_{0}} .\end{cases}
$$

In [16], L. Escauriaza gave an example to show that the above system has a nonzero solution when $\theta_{0}<\frac{\pi}{2}$ and the result of $\mathrm{Lu} \mathrm{Li}$ and V. Šverák implied that the system has only zero solution when $\pi>\theta_{0}>2 \arccos (1 / \sqrt{3}) \approx 109.5^{\circ}$. Now we consider a cone of dimension 2,

$$
\mathcal{C}_{\theta_{0}}=\left\{(r, \theta) \mid 0<\theta<\theta_{0}\right\},\left(0<\theta_{0}<\pi\right)
$$


and $u\left(x_{1}, x_{2}, t\right)$ is the solution of system (8) in dimension 2 , where

$$
\left\{\begin{array}{l}
x_{1}=r \cos \theta \\
x_{2}=r \sin \theta
\end{array}\right.
$$

Let

$$
\bar{\theta}=l \theta, \text { with } l=\frac{\pi}{\theta_{0}}>1,
$$

and

$$
\left\{\begin{array}{l}
y_{1}=r \cos \bar{\theta} \\
y_{2}=r \sin \bar{\theta}
\end{array}\right.
$$

then $\left(y_{1}, y_{2}\right) \in \mathbb{R}_{+}^{2}$. We denote

$$
v\left(y_{1}, y_{2}, t\right)=u\left(x_{1}, x_{2}, t\right),\left(y_{1}, y_{2}, t\right) \in \mathbb{R}_{+}^{2} \times[0, T] .
$$

By simple calculation,

$$
\begin{aligned}
\partial_{x_{1}}^{2}+\partial_{x_{2}}^{2}= & \partial_{r}^{2}+\frac{\partial_{r}}{r}+\frac{\partial_{\theta}^{2}}{r^{2}}=\partial_{r}^{2}+\frac{\partial_{r}}{r}+l^{2} \frac{\partial_{\bar{\theta}}^{2}}{r^{2}} \\
= & \left(\partial_{r}^{2}+\frac{\partial_{r}}{r}+\frac{\partial_{\bar{\theta}}^{2}}{r^{2}}\right)+\left(l^{2}-1\right) \frac{\partial_{\bar{\theta}}^{2}}{r^{2}} \\
= & \partial_{y_{1}}^{2}+\partial_{y_{2}}^{2}+\left(l^{2}-1\right)\left(\frac{y_{2}^{2}}{r^{2}} \partial_{y_{1}}^{2}+\frac{y_{1}^{2}}{r^{2}} \partial_{y_{2}}^{2}-2 \frac{y_{1} y_{2}}{r^{2}} \partial_{y_{1} y_{2}}-\frac{y_{1}}{r^{2}} \partial_{y_{1}}-\frac{y_{2}}{r^{2}} \partial_{y_{2}}\right) \\
= & {\left[1+\left(l^{2}-1\right) \frac{y_{2}^{2}}{r^{2}}\right] \partial_{y_{1}}^{2}+\left[1+\left(l^{2}-1\right) \frac{y_{1}^{2}}{r^{2}}\right] \partial_{y_{2}}^{2} } \\
& -2\left(l^{2}-1\right) \frac{y_{1} y_{2}}{r^{2}} \partial_{y_{1} y_{2}}-\left(l^{2}-1\right)\left(\frac{y_{1}}{r^{2}} \partial_{y_{1}}+\frac{y_{2}}{r^{2}} \partial_{y_{2}}\right) \\
= & \nabla \cdot(A \nabla v),
\end{aligned}
$$

where

$$
A\left(y_{1}, y_{2}\right)=\left(\begin{array}{cc}
1+\left(l^{2}-1\right) \frac{y_{2}^{2}}{r^{2}} & -\left(l^{2}-1\right) \frac{y_{1} y_{2}}{r^{2}} \\
-\left(l^{2}-1\right) \frac{y_{1} y_{2}}{r^{2}} & 1+\left(l^{2}-1\right) \frac{y_{1}^{2}}{r^{2}}
\end{array}\right) .
$$

Together with the equation in (8) we can deduce that

$$
\partial_{t} v+\nabla \cdot(A \nabla v)=0
$$

and $A$ is positive since $l>1$.

Denote

$$
w\left(y_{1}, y_{2}, t\right)=v\left(y_{1}, y_{2}+1, t\right),\left(y_{1}, y_{2}, t\right) \in \mathbb{R}_{+}^{2} \times[0, T],
$$

and

$$
B\left(y_{1}, y_{2}\right)=A\left(y_{1}, y_{2}+1\right) \equiv\left(\begin{array}{ll}
b^{11} & b^{12} \\
b^{21} & b^{22}
\end{array}\right)
$$

Direct calculations give us

$$
\left|\nabla b^{i j}\right| \leq l^{2}-1 \text { and }\left|\nabla b^{i j}\right| \leq \frac{l^{2}-1}{r} \equiv \frac{E_{1}}{r} .
$$

By (10) we have

$$
\partial_{t} w+\nabla \cdot(B \nabla w)=0
$$


By the notations of $u$ and $w$, we see that $w$ is a solution of the following system:

$$
\begin{cases}\partial_{t} w+\nabla \cdot(B \nabla w)=0 & \text { in } \mathbb{R}_{+}^{2} \times[0, T], \\ |w(y, t)| \leq N & \text { in } \mathbb{R}_{+}^{2} \times[0, T], \\ w(y, 0)=0 & \text { in } \mathbb{R}_{+}^{2} .\end{cases}
$$

By the result of Li and Šverák [16], we conclude that when

$$
E_{1}<\left(\frac{\pi}{2 \arccos (1 / \sqrt{3})}\right)^{2}-1
$$

we have

$$
1<l<\frac{\pi}{2 \arccos (1 / \sqrt{3})}, \quad 2 \arccos (1 / \sqrt{3})<\theta_{0}<\pi
$$

and then $u \equiv 0$ and thus $w \equiv 0$.

When $E_{1}>3$, we have $l>2, \theta_{0}<\frac{\pi}{2}$, and then (8) has a nonzero solution and thus (11) must also has a nonzero solution $w$. Otherwise, if $w \equiv 0$, this means that $u=0$ in $D \times[0, T]$, where $D$ is a certain subregion of $\mathcal{C}_{\theta_{0}}$. Then by the unique continuation result, we have $u \equiv 0$, which contradicts that $u$ is nonzero. In this case BU fails, although $\left|\nabla b^{i j}\right|$ are bounded and $\left|\nabla b^{i j}\right| \leq \frac{E_{1}}{r}$.

The example above shows that the decay at infinity conditions, those are assumptions in (4), where $E$ is less than a given constant, are optimal in some sense.

To prove Theorem 1.1 we need to obtain the corresponding Carleman inequalities. Now we introduce two Carleman inequalities for the case of variable coefficients. They are generalizations of the two Carleman inequalities for the case of constant coefficients, as shown in [8, 9].

Proposition 1.3. Suppose $\left\{a^{i j}\right\}$ satisfy (1) and

$$
\left|\nabla_{x} a^{i j}(x, t)\right|+\left|\partial_{t} a^{i j}(x, t)\right| \leq M,\left|\nabla_{x} a^{i j}(x, t)\right| \leq \frac{E}{|x|}, \quad \forall(x, t) \in \mathbb{R}^{n} \times(0,2) .
$$

Then there exists a constant $K=K(n, \Lambda, \lambda, M, E)$, such that for any $u \in C_{0}^{\infty}\left(\mathbb{R}^{n} \times(0,2)\right)$ and any number $\gamma>0$,

$$
\begin{array}{r}
\int_{\mathbb{R}^{n} \times(0,2)} e^{2 \gamma\left(t^{-K}-1\right)-\frac{b|x|^{2}+K}{t}}\left(|u|^{2}+|\nabla u|^{2}\right) d x d t \\
\leq \int_{\mathbb{R}^{n} \times(0,2)} e^{2 \gamma\left(t^{-K}-1\right)-\frac{b|x|^{2}+K}{t}}|P u|^{2} d x d t
\end{array}
$$

where $b=\frac{1}{8 \Lambda}$.

Proposition 1.4. Suppose $\left\{a^{i j}\right\}$ satisfy (1) and

$$
\left|\nabla_{x} a^{i j}(x, t)\right|+\left|\partial_{t} a^{i j}(x, t)\right| \leq M,\left|\nabla_{x} a^{i j}(x, t)\right| \leq \frac{E}{|x|}, \quad \forall(x, t) \in \mathbb{R}_{+}^{n} \times(0,1) .
$$

Let $Q=\left\{(x, t) \mid x_{n} \geq 1, t \in(0,1)\right\}$ and

$$
\psi(x)=|x|^{2}-2 \frac{\Lambda}{\lambda}|x| x_{n}+2\left(\frac{\Lambda}{\lambda}\right)^{2} x_{n}^{2} .
$$

Then there exist positive constants $E_{0}=E_{0}(n, \Lambda, \lambda), \alpha=\alpha(n, \Lambda, \lambda, E) \in(1,2), b=$ $b(\Lambda, \lambda)$ and $K=K(n, \Lambda, \lambda, M, E)$ such that when $E<E_{0}$, for any function $u \in C_{0}^{\infty}(Q)$ 
and any number $\gamma>0$, we have

$$
\begin{gathered}
\int_{Q} e^{2 \gamma\left(t^{-K}-1\right) x_{n}^{\alpha}-\frac{b \psi(x)+K}{t}}\left(|u|^{2}+|\nabla u|^{2}\right) d x d t \\
\leq \int_{Q} e^{2 \gamma\left(t^{-K}-1\right) x_{n}^{\alpha}-\frac{b \psi(x)+K}{t}}|P u|^{2} d x d t
\end{gathered}
$$

Remark 1.5. In fact, we can take $E_{0}=\frac{\lambda}{16 n^{2} \frac{\Lambda}{\lambda}\left(\frac{\Lambda}{\lambda}+1\right)}, \alpha=1+\frac{E}{E_{0}}$ and $b=\frac{1}{64 \Lambda\left(\frac{\Lambda}{\lambda}+1\right)^{4}}$ in Proposition 1.4 which can be seen from the proof.

Carleman inequality (13) is the key results in this paper. Assuming it, there is only a standard argument by following the corresponding parts of Escauriaza, Seregin, and Šverák in [7, 8] to prove Theorem 1.1. In the establishment of Carleman inequality (13), the construction of the function $\psi$ is crucial.

Remark 1.6. It is worthwhile to note that Carleman inequality (12) does not require the smallness of E, while Carleman inequality (13) does, which is stronger.

Moreover, the Carleman inequality (13) for the parabolic operators with variable coefficients in a half space is stronger than the one for the case of constant coefficients, as shown in [8, 9]. When $P$ is the backward heat operator, there are two Carleman inequalities to prove BU. The first one implies an exponential decay of the solution, which enable us to apply the second one to prove BU. And here, we just need one Carleman inequality (13) to prove BU. We list Carleman inequality (12) here just for comparison with the case of constant coefficients.

The paper organized as follows. We first make use of Carleman inequality (13) to prove Theorem 1.1 in next section. Then we prove the two Carleman inequalities Proposition 1.3 and Proposition 1.4 in the last section.

\section{Proof of Theorem 1.1}

In this section, we prove the main theorem by assuming Proposition 1.4 first. Then we shall prove the Carleman inequalities in next section.

We always assume that $T=1$ and extend $u$ and $a^{i j}$ by the following way:

$$
\begin{gathered}
u(x, t)=0, \text { if } t<0 ; \\
a^{i j}(x, t)=a^{i j}(x, 0) \text {, if } t<0 .
\end{gathered}
$$

We denote $e_{n}=(0,0, \ldots, 0,1)$.

The next lemma implies Theorem 1.1 immediately.

Lemma 2.1. Suppose $\left\{a^{i j}\right\}$ and $u$ satisfy assumptions (1), (3)-(5). Then there exists $T_{1}=T_{1}(\Lambda, \lambda, N) \in\left(0, \frac{1}{2}\right)$, such that

$$
u(x, t) \equiv 0
$$

in $\mathbb{R}_{+}^{n} \times\left(0, T_{1}\right)$.

Proof. We make use of Carleman inequality (13) to prove this lemma. We mainly follow the arguments of corresponding parts of Escauriaza, Seregin and Šverák in [7, 8]. By the regularity theory for solutions of parabolic equations, we have

$$
|u(x, t)|+|\nabla u(x, t)| \leq C(n, \Lambda, \lambda, M, N) e^{2 N|x|^{2}}
$$



for $(x, t) \in\left(\mathbb{R}_{+}^{n}+e_{n}\right) \times\left(0, \frac{1}{2}\right)$. Let

$$
T_{1}=\min \left\{\frac{b}{32 N}, \frac{1}{12 N^{2}}, \frac{1}{2}\right\},
$$

where $b$ is the one in Proposition 1.4. $\operatorname{Let} \tau=\sqrt{2 T_{1}}$.

We denote

$$
v(y, s)=u\left(\tau y, \tau^{2} s-T_{1}\right)
$$

and

$$
\tilde{a}^{i j}(y, s)=a^{i j}\left(\tau y, \tau^{2} s-T_{1}\right)
$$

for $(y, s) \in \mathbb{R}_{+}^{n} \times(0,1)$. Then it is easy to see

$$
\left|\nabla_{y} \tilde{a}^{i j}(y, s)\right|+\left|\partial_{s} \tilde{a}^{i j}(y, s)\right| \leq \tau M \leq M,
$$

and

$$
\left|\nabla_{y} \tilde{a}^{i j}(y, s)\right|=\tau\left|\nabla a^{i j}\left(\tau y, \tau^{2} s-T_{1}\right)\right| \leq \tau \frac{E}{|\tau y|}=\frac{E}{|y|} .
$$

We denote

$$
\tilde{P} v=\partial_{s} v+\sum_{i j} \partial_{i, y}\left(\tilde{a}^{i j} \partial_{j, y} v\right)
$$

by our notation and (5),

$$
|\tilde{P} v| \leq \tau N(|v|+|\nabla v|),
$$

for $(y, s) \in \mathbb{R}_{+}^{n} \times(0,1)$. From (14), we have

$$
|v(y, s)|+|\nabla v(y, s)| \leq C(n, \Lambda, \lambda, M, N) e^{2 N \tau^{2}|y|^{2}}
$$

for $(y, s) \in\left(\mathbb{R}_{+}^{n}+\frac{1}{\tau} e_{n}\right) \times(0,1)$; and

$$
v(y, s)=0
$$

for $(y, s) \in \mathbb{R}_{+}^{n} \times\left(0, \frac{1}{2}\right]$.

In order to apply Carleman inequality (13), we choose two smooth cut-off functions such that

$$
\eta_{1}(p)= \begin{cases}0, & \text { if } p<\frac{1}{\tau}+1 \\ 1, & \text { if } p>\frac{1}{\tau}+2\end{cases}
$$

And

$$
\eta_{2}(q)= \begin{cases}0, & \text { if } q<-\frac{3}{4} \\ 1, & \text { if } q>-\frac{1}{2}\end{cases}
$$

All functions take values in $[0,1]$ and $\left|\eta_{1}^{\prime}\right|,\left|\eta_{1}^{\prime \prime}\right|,\left|\eta_{2}^{\prime}\right|$ and $\left|\eta_{2}^{\prime \prime}\right|$ are all bounded. Denote

$$
f(s)=s^{-K}-1
$$

and

$$
C_{\star}=1+\sup _{\substack{\frac{1}{2}<s<1 \\ \frac{1}{\tau}+1<y_{n}<\frac{1}{\tau}+2}}\left\{f(s) y_{n}^{\alpha}\right\}=1+f\left(\frac{1}{2}\right)\left(\frac{1}{\tau}+2\right)^{\alpha} .
$$

Set

$$
\eta(y, s)=\eta_{1}\left(y_{n}\right) \eta_{2}\left(\frac{f(s) y_{n}^{\alpha}}{2 C_{\star}}-1\right),
$$


and $w=\eta v$. Then supp $w \subset Q$, and

$$
\begin{aligned}
|\tilde{P} w| & =\left|\eta \tilde{P} v+v \tilde{P} \eta+2 \tilde{a}^{i j} \partial_{i} \eta \partial_{j} v\right| \\
& \leq|\eta \tilde{P} v|+C(n, \Lambda, M) \chi(|v|+|\nabla v|)\left(\left|\partial_{s} \eta\right|+|\nabla \eta|+\left|\nabla^{2} \eta\right|\right),
\end{aligned}
$$

where $\chi$ is the characteristic function of the set

$$
\Omega=\left\{(y, s) \mid \frac{1}{2}<s<1,0<\eta<1\right\} .
$$

By (16), we have

$$
\begin{aligned}
|\tilde{P} w| & \leq \eta \tau N(|v|+|\nabla v|)+C(n, \Lambda, M) \chi(|v|+|\nabla v|)\left(\left|\partial_{s} \eta\right|+|\nabla \eta|+\left|\nabla^{2} \eta\right|\right) \\
& \leq \tau N(|w|+|\nabla w|)+C(n, \Lambda, M, N) \chi(|v|+|\nabla v|)\left(\left|\partial_{s} \eta\right|+|\nabla \eta|+\left|\nabla^{2} \eta\right|\right) .
\end{aligned}
$$

Notice that $\frac{1}{2}<s<1$ in $\Omega$, and when $\frac{1}{2}<s<1$,

$$
\left|\partial_{s} \eta\right|+|\nabla \eta|+\left|\nabla^{2} \eta\right| \leq C(n, \Lambda, \lambda, M, E) y_{n}^{\alpha} \leq C(n, \Lambda, \lambda, M, E) y_{n}^{2},
$$

then

$$
|\tilde{P} w| \leq \tau N(|w|+|\nabla w|)+C(n, \Lambda, \lambda, M, E, N) \chi(|v|+|\nabla v|) y_{n}^{2}
$$

Moreover,

$$
\begin{aligned}
\Omega= & \left\{(y, s) \mid \frac{1}{2}<s<1, \eta_{1}>0,0<\eta_{2}<1\right\} \\
& \bigcup\left\{(y, s) \mid \frac{1}{2}<s<1,0<\eta_{1}<1, \eta_{2}=1\right\} \\
= & \left\{(y, s) \mid \frac{1}{2}<s<1, y_{n}>\frac{1}{\tau}+1, \frac{1}{2}<\frac{f(s) y_{n}^{\alpha}}{C_{\star}}<1\right\} \\
& \bigcup\left\{(y, s) \mid \frac{1}{2}<s<1, \frac{1}{\tau}+1<y_{n}<\frac{1}{\tau}+2, \frac{f(s) y_{n}^{\alpha}}{C_{\star}} \geq 1\right\} .
\end{aligned}
$$

By the choice of $C_{\star}$ we obtain that the second set of the right side of the above identity is empty, then

$$
\Omega=\left\{(y, s) \mid \frac{1}{2}<s<1, y_{n}>\frac{1}{\tau}+1, \frac{1}{2}<\frac{f(s) y_{n}^{\alpha}}{C_{\star}}<1\right\} .
$$

By (17), in the support of $w$ we have

$$
e^{2 \gamma f(s) y_{n}^{\alpha}-\frac{b \psi(y)+K}{s}}(|v|+|\nabla v|)^{2} \leq C e^{2 \gamma f(s) y_{n}^{\alpha}-\frac{b \psi(y)+K}{s}+4 N \tau^{2}|y|^{2}} .
$$

Notice that $\psi(y) \geq \frac{|y|^{2}}{2}$ and $\tau=\sqrt{2 T_{1}}$, then we have

$$
e^{2 \gamma f(s) y_{n}^{\alpha}-\frac{b \psi(y)+K}{s}}(|v|+|\nabla v|)^{2} \leq C e^{2 \gamma f(s) y_{n}^{\alpha}-\frac{b}{2}|y|^{2}+8 N T_{1}|y|^{2}} .
$$

By (15), we know that $T_{1} \leq \frac{b}{32 N}$, then in supp $w$ we have

$$
e^{2 \gamma f(s) y_{n}^{\alpha}-\frac{b \psi(y)+K}{s}}(|v|+|\nabla v|)^{2} \leq C e^{2 \gamma f(s) y_{n}^{\alpha}-\frac{b}{4}|y|^{2}} .
$$


Although supp $w$ may be unbounded, supp $w \subset Q$ and (21) allow us to claim the validity of Proposition 1.4 for $w$. Then by Carleman inequality (13), together with (19), we have

$$
\begin{aligned}
J & \equiv \int_{Q} e^{2 \gamma f(s) y_{n}^{\alpha}-\frac{b \psi(y)+K}{s}}\left(|w|^{2}+|\nabla w|^{2}\right) d y d s \\
& \leq \int_{Q} e^{2 \gamma f(s) y_{n}^{\alpha}-\frac{b \psi(y)+K}{s}}|\tilde{P} w|^{2} d y d s \\
& \leq 3 \tau^{2} N^{2} J+C \int_{Q} e^{2 \gamma f(s) y_{n}^{\alpha}-\frac{b \psi(y)+K}{s}} \chi(|v|+|\nabla v|)^{2} y_{n}^{4} d y d s .
\end{aligned}
$$

By (15), we know that $3 \tau^{2} N^{2}=6 T_{1} N^{2} \leq \frac{1}{2}$. We deduce from the above inequality that

$$
J \leq C \int_{\Omega} e^{2 \gamma f(s) y_{n}^{\alpha}-\frac{b \psi(y)+K}{s}}(|v|+|\nabla v|)^{2} y_{n}^{4} d y d s
$$

By (21), we have

$$
J \leq C \int_{\Omega} e^{2 \gamma f(s) y_{n}^{\alpha}-\frac{b}{4}|y|^{2}} y_{n}^{4} d y d s .
$$

By (20) we have that $f(s) y_{n}^{\alpha}<C_{\star}$ in $\Omega$, then

$$
J \leq C e^{2 \gamma C_{\star}} \int_{\Omega} e^{-\frac{b}{4}|y|^{2}} y_{n}^{4} d y d s \leq C e^{2 \gamma C_{\star}} .
$$

On the other hand, we denote

$$
\begin{gathered}
\Omega_{1}=\{(y, s) \mid 0<s<1, \eta=1\}=\left\{(y, s) \mid 0<s<1, y_{n} \geq \frac{1}{\tau}+2, \frac{f(s) y_{n}^{\alpha}}{C_{\star}} \geq 1\right\}, \\
\Omega_{2}=\left\{(y, s) \mid 0<s<1, y_{n} \geq \frac{1}{\tau}+2, \frac{f(s) y_{n}^{\alpha}}{C_{\star}} \geq 2\right\} .
\end{gathered}
$$

Obviously $\Omega_{2} \subset \Omega_{1}$ and $w=v$ in $\Omega_{1}$. Then

$$
\begin{aligned}
J & \geq \int_{\Omega_{1}} e^{2 \gamma f(s) y_{n}^{\alpha}-\frac{b \psi(y)+K}{s}}\left(|v|^{2}+|\nabla v|^{2}\right) d y d s \\
& \geq \int_{\Omega_{2}} e^{2 \gamma f(s) y_{n}^{\alpha}-\frac{b \psi(y)+K}{s}}\left(|v|^{2}+|\nabla v|^{2}\right) d y d s
\end{aligned}
$$

By (23), we know that $f(s) y_{n}^{\alpha} \geq 2 C_{\star}$ in $\Omega_{2}$. Hence

$$
J \geq e^{4 \gamma C_{\star}} \int_{\Omega_{2}} e^{-\frac{b \psi(y)+K}{s}}\left(|v|^{2}+|\nabla v|^{2}\right) d y d s .
$$

Combining (22) and (24), we have

$$
\int_{\Omega_{2}} e^{-\frac{b \psi(y)+K}{s}}\left(|v|^{2}+|\nabla v|^{2}\right) d y d s \leq C e^{-2 \gamma C_{\star}} .
$$

Passing to the limit as $\gamma \rightarrow+\infty$, we obtain $v(y, s)=0$ in $\Omega_{2}$. Using unique continuation though spatial boundaries (see [10]), we obtain that $v(y, s) \equiv 0$ in $\mathbb{R}_{+}^{n} \times(0,1)$. That is, $u(x, t) \equiv 0$ in $\mathbb{R}_{+}^{n} \times\left(0, T_{1}\right)$. Thus we proved this lemma. 


\section{Proof of Carleman Inequalities}

In this section, we shall prove two Carleman Inequalities which is the crucial part of the whole argument. The main idea is to choose a proper weight function $G$. We denote

$$
\tilde{\Delta} u=\partial_{i}\left(a^{i j} \partial_{j} u\right) \text {. }
$$

Here and in the following argument, we use the summation convention on the repeated indices. We shall make use of the following lemma which is due to L. Escauriaza and F. J. Fernández in [10] (see also [18]).

Lemma 3.1. Suppose $\sigma(t): \mathbb{R}_{+} \rightarrow \mathbb{R}_{+}$is a smooth function, $\alpha$ is a real number, $F$ and $G$ are differentiable functions and $G>0$. Then the following identity holds for any $u \in C_{0}^{\infty}\left(\mathbb{R}^{n} \times(0, T)\right)$

$$
\begin{aligned}
& 2 \int_{\mathbb{R}^{n} \times(0, T)} \frac{\sigma^{1-\alpha}}{\sigma^{\prime}}|L u|^{2} G d x d t+\frac{1}{2} \int_{\mathbb{R}^{n} \times(0, T)} \frac{\sigma^{1-\alpha}}{\sigma^{\prime}} u^{2} M G d s d t \\
& +\int_{\mathbb{R}^{n} \times(0, T)} \frac{\sigma^{1-\alpha}}{\sigma^{\prime}}\langle A \nabla u, \nabla u\rangle\left[\left(\log \frac{\sigma}{\sigma^{\prime}}\right)^{\prime}+\frac{\partial_{t} G-\tilde{\Delta} G}{G}-F\right] G d x d t \\
& +2 \int_{\mathbb{R}^{n} \times(0, T)} \frac{\sigma^{1-\alpha}}{\sigma^{\prime}}\left\langle D_{G} \nabla u, \nabla u\right\rangle G d x d t-\int_{\mathbb{R}^{n} \times(0, T)} \frac{\sigma^{1-\alpha}}{\sigma^{\prime}} u\langle A \nabla u, \nabla F\rangle G d x d t \\
& =2 \int_{\mathbb{R}^{n} \times(0, T)} \frac{\sigma^{1-\alpha}}{\sigma^{\prime}} L u P u G d x d t
\end{aligned}
$$

where

and

$$
\begin{gathered}
L u=\partial_{t} u-\langle A \nabla \log G, \nabla u\rangle+\frac{F u}{2}-\frac{\alpha \sigma^{\prime}}{2 \sigma} u, \\
M=\left(\log \frac{\sigma}{\sigma^{\prime}}\right)^{\prime} F+\partial_{t} F+\left(F-\alpha \frac{\sigma^{\prime}}{\sigma}\right)\left(\frac{\partial_{t} G-\tilde{\Delta} G}{G}-F\right)-\langle A \nabla F, \nabla \log G\rangle,
\end{gathered}
$$

$$
D_{G}^{i j}=a^{i k} \partial_{k l}(\log G) a^{l j}+\frac{\partial_{l}(\log G)}{2}\left(a^{k i} \partial_{k} a^{l j}+a^{k j} \partial_{k} a^{l i}-a^{k l} \partial_{k} a^{i j}\right)+\frac{1}{2} \partial_{t} a^{i j} .
$$

We first give a modification of this lemma which will be used in our proof. Letting $\alpha=0$ and $\sigma(t)=e^{t}$ in Lemma 3.1, we obtain the following identity for $u \in C_{0}^{\infty}\left(\mathbb{R}^{n} \times(0, T)\right)$

$$
\begin{aligned}
& \frac{1}{2} \int_{\mathbb{R}^{n} \times(0, T)} u^{2} M G d x d t+\int_{\mathbb{R}^{n} \times(0, T)}\left[2\left\langle D_{G} \nabla u, \nabla u\right\rangle+\langle A \nabla u, \nabla u\rangle\left(\frac{\partial_{t} G-\tilde{\Delta} G}{G}-F\right)\right] G d x d t \\
& -\int_{\mathbb{R}^{n} \times(0, T)} u\langle A \nabla u, \nabla F\rangle G d x d t=2 \int_{\mathbb{R}^{n} \times(0, T)} L u(P u-L u) G d x d t .
\end{aligned}
$$

If $\nabla F$ is differentiable, we can integrate by parts to obtain

$$
\begin{aligned}
& -\int_{\mathbb{R}^{n} \times(0, T)} u\langle A \nabla u, \nabla F\rangle G d x d t \\
= & \frac{1}{2} \int_{\mathbb{R}^{n} \times(0, T)} u^{2} \tilde{\Delta} F G d x d t+\frac{1}{2} \int_{\mathbb{R}^{n} \times(0, T)} u^{2}\langle A \nabla F, \nabla \log G\rangle G d x d t .
\end{aligned}
$$

The function $\nabla F$ may not be differentiable, so we approximate $F$ by some smooth function $F_{0}$ and use the above identity with $F_{0}$ in place of $F$, following Tu's idea in [18]. Thus we obtain the following result. 
BACKWARD UNIQUENESS FOR PARABOLIC OPERATORSWITH VARIABLE COEFFICIENTS IN A HALF SPAGE

Corollary 3.2. Suppose $F$ and $G$ are differentiable functions and $G>0$. Then the following identity holds for any $u \in C_{0}^{\infty}\left(\mathbb{R}^{n} \times(0, T)\right)$

$$
\begin{aligned}
& \frac{1}{2} \int_{\mathbb{R}^{n} \times(0, T)} u^{2} M_{0} G d x d t+\int_{\mathbb{R}^{n} \times(0, T)}\left[2\left\langle D_{G} \nabla u, \nabla u\right\rangle+\langle A \nabla u, \nabla u\rangle\left(\frac{\partial_{t} G-\tilde{\Delta} G}{G}-F\right)\right] G d x d t \\
& -\int_{\mathbb{R}^{n} \times(0, T)} u\left\langle A \nabla u, \nabla\left(F-F_{0}\right)\right\rangle G d x d t=2 \int_{\mathbb{R}^{n} \times(0, T)} L u(P u-L u) G d x d t,
\end{aligned}
$$

where

$$
\begin{gathered}
L u=\partial_{t} u-\langle A \nabla u, \nabla \log G\rangle+\frac{F u}{2}, \\
M_{0}=\partial_{t} F+F\left(\frac{\partial_{t} G-\tilde{\Delta} G}{G}-F\right)+\tilde{\Delta} F_{0}-\left\langle A \nabla\left(F-F_{0}\right), \nabla \log G\right\rangle,
\end{gathered}
$$

and$$
D_{G}^{i j}=a^{i k} \partial_{k l}(\log G) a^{l j}+\frac{\partial_{l}(\log G)}{2}\left(a^{k i} \partial_{k} a^{l j}+a^{k j} \partial_{k} a^{l i}-a^{k l} \partial_{k} a^{i j}\right)+\frac{1}{2} \partial_{t} a^{i j} .
$$

3.1. Proof of Proposition 1.3. We use identity (26) to prove Proposition 1.3, In (26), we let

$$
G=e^{2 \gamma\left(t^{-K}-1\right)-\frac{b|x|^{2}+K}{t}}
$$

then

$$
\frac{\partial_{t} G-\tilde{\Delta} G}{G}=\frac{b|x|^{2}-4 b^{2} a^{i j} x_{i} x_{j}+K}{t^{2}}+\frac{2 b a^{i i}+2 b \partial_{k} a^{k l} x_{l}}{t}-2 \gamma K t^{-K-1} .
$$

Let

$$
F=\frac{b|x|^{2}-4 b^{2} a^{i j} x_{i} x_{j}+K}{t^{2}}+\frac{2 b a^{i i}}{t}-2 \gamma K t^{-K-1}-d\left(\frac{1}{t}+1\right),
$$

where $d$ is a positive constant to be determined. Set

$$
F_{0}=\frac{b|x|^{2}-4 b^{2} a_{\epsilon}^{i j} x_{i} x_{j}+K}{t^{2}}+\frac{2 b a_{\epsilon}^{i i}}{t}-2 \gamma K t^{-K-1}-d\left(\frac{1}{t}+1\right),
$$

where

$$
a_{\epsilon}^{i j}(x, t)=\int_{\mathbb{R}^{n}} a^{i j}(x-y, t) \phi_{\epsilon}(y) d y,
$$

$\phi$ is a mollifier, and $\epsilon=\frac{1}{2}$.

We denote by $I_{n}$ the identity matrix of $\mathbb{R}^{n}, C$ are generic constants depending on $n, \Lambda, \lambda, M$ and $E$ in the following arguments. We need some estimates which we list in the following lemma.

Lemma 3.3. Set $b=\frac{1}{8 \Lambda}$ and $K=12 d$. For $d=d(n, \Lambda, \lambda, M, E)$ large enough, we have

$$
\begin{gathered}
2 D_{G}+A\left(\frac{\partial_{t} G-\tilde{\Delta} G}{G}-F\right) \geq\left(\frac{1}{t}+1\right) I_{n} ; \\
\partial_{t} F+F\left(\frac{\partial_{t} G-\tilde{\Delta} G}{G}-F\right) \geq \frac{d b\left(|x|^{2}+1\right)}{4 t^{3}} ; \\
\tilde{\triangle} F_{0} \geq-\frac{C\left(|x|^{2}+1\right)}{t^{3}}
\end{gathered}
$$




$$
\left|\nabla\left(F-F_{0}\right)\right| \leq \frac{C(|x|+1)}{t^{2}} .
$$

We will prove this lemma later.

First by applying (27) we have

$$
\begin{aligned}
& \int_{\mathbb{R}^{n} \times(0,2)}\left[2\left\langle D_{G} \nabla u, \nabla u\right\rangle+\langle A \nabla u, \nabla u\rangle\left(\frac{\partial_{t} G-\tilde{\Delta} G}{G}-F\right)\right] G d x d t \\
\geq & \int_{\mathbb{R}^{n} \times(0,2)}\left(\frac{1}{t}+1\right)|\nabla u|^{2} G d x d t .
\end{aligned}
$$

Next we estimate $M_{0}$. By applying (30) we have

$$
\left|\left\langle A \nabla\left(F-F_{0}\right), \nabla \log G\right\rangle\right| \leq \Lambda\left|\nabla\left(F-F_{0}\right)\right||\nabla \log G| \leq \frac{C(|x|+1)|x|}{t^{3}} \leq \frac{C\left(|x|^{2}+1\right)}{t^{3}} .
$$

Then by (28), (29) and (32) we have

$$
\begin{aligned}
M_{0} & =\partial_{t} F+F\left(\frac{\partial_{t} G-\tilde{\Delta} G}{G}-F\right)+\tilde{\Delta} F_{0}-\left\langle A \nabla\left(F-F_{0}\right), \nabla \log G\right\rangle \\
& \geq\left(\frac{d b}{4}-C\right) \frac{|x|^{2}+1}{t^{3}},
\end{aligned}
$$

thus

$$
\frac{1}{2} \int_{\mathbb{R}^{n} \times(0,2)} u^{2} M_{0} G d x d t \geq\left(\frac{d b}{8}-C\right) \int_{\mathbb{R}^{n} \times(0,2)} \frac{|x|^{2}+1}{t^{3}} u^{2} G d x d t .
$$

By (30) and the Cauchy inequality we have

$$
\begin{aligned}
& \left|\int_{\mathbb{R}^{n} \times(0,2)} u\left\langle A \nabla u, \nabla\left(F-F_{0}\right)\right\rangle G d x d t\right| \\
\leq & \Lambda \int_{\mathbb{R}^{n} \times(0,2)}\left|\nabla\left(F-F_{0}\right)\right||u||\nabla u| G d x d t \\
\leq & C \int_{\mathbb{R}^{n} \times(0,2)} \frac{|x|+1}{t^{2}}|u||\nabla u| G d x d t \\
\leq & C \int_{\mathbb{R}^{n} \times(0,2)} \frac{|x|^{2}+1}{t^{3}}|u|^{2} G d x d t+\int_{\mathbb{R}^{n} \times(0,2)} \frac{|\nabla u|^{2}}{t} G d x d t .
\end{aligned}
$$

Finally, by (26), (31), (33), (34) and the Cauchy inequality, we have

$$
\int_{\mathbb{R}^{n} \times(0,2)}|P u|^{2} G d x d t \geq\left(\frac{d b}{8}-C\right) \int_{\mathbb{R}^{n} \times(0,2)} \frac{|x|^{2}+1}{t^{3}}|u|^{2} G d x d t+\int_{\mathbb{R}^{n} \times(0,2)}|\nabla u|^{2} G d x d t,
$$

if we choose $d$ large enough, we obtain

$$
\int_{\mathbb{R}^{n} \times(0,2)}|P u|^{2} G d x d t \geq \int_{\mathbb{R}^{n} \times(0,2)}\left(|u|^{2}+|\nabla u|^{2}\right) G d x d t .
$$

Thus we proved Carleman inequality (12).

Proof of Lemma 3.3. We estimate them one by one. 
Estimate of $2 D_{G}+A\left(\frac{\partial_{t} G-\tilde{\Delta} G}{G}-F\right)$.

By direct calculations we have

$$
\begin{aligned}
& 2 D_{G}+A\left(\frac{\partial_{t} G-\tilde{\Delta} G}{G}-F\right) \\
= & -\frac{4 b}{t} A^{2}-\frac{2 b x_{l}}{t}\left(a^{k i} \partial_{k} a^{l j}+a^{k j} \partial_{k} a^{l i}-a^{k l} \partial_{k} a^{i j}\right)+\partial_{t} a^{i j}+A\left(\frac{d+2 b \partial_{k} a^{k l} x_{l}}{t}+d\right) \\
= & -\frac{4 b}{t} A^{2}-\frac{2 b x_{l}}{t}\left(a^{k i} \partial_{k} a^{l j}+a^{k j} \partial_{k} a^{l i}-a^{k l} \partial_{k} a^{i j}-a^{i j} \partial_{k} a^{k l}\right)+\partial_{t} a^{i j}+A d\left(\frac{1}{t}+1\right) \\
\geq & -\frac{4 b}{t} \Lambda^{2} I_{n}-\frac{2 b x_{l}}{t}\left(a^{k i} \partial_{k} a^{l j}+a^{k j} \partial_{k} a^{l i}-a^{k l} \partial_{k} a^{i j}-a^{i j} \partial_{k} a^{k l}\right)+\partial_{t} a^{i j}+\lambda d\left(\frac{1}{t}+1\right) I_{n} .
\end{aligned}
$$

Next we estimate the lower bounds of the matrices in the right side of the above inequality.

We just need to estimate matrix $x_{l} a^{k i} \partial_{k} a^{l j}$ and $\partial_{t} a^{i j}$. For any $\xi \in \mathbb{R}^{n}$,

$$
\left|x_{l} a^{k i} \partial_{k} a^{l j} \xi_{i} \xi_{j}\right| \leq n^{2}|x| \Lambda \frac{E}{|x|} \sum_{i, j}\left|\xi_{i}\right|\left|\xi_{j}\right| \leq n^{3} \Lambda E|\xi|^{2},
$$

then

$$
-n^{3} \Lambda E I_{n} \leq x_{l} a^{k i} \partial_{k} a^{l j} \leq n^{3} \Lambda E I_{n}
$$

and

$$
\left|\partial_{t} a^{i j} \xi_{i} \xi_{j}\right| \leq M \sum_{i, j}\left|\xi_{i}\right|\left|\xi_{j}\right| \leq M n|\xi|^{2}
$$

then

$$
\partial_{t} a^{i j} \geq-M n I_{n}
$$

Thus we have

$$
2 D_{G}+A\left(\frac{\partial_{t} G-\tilde{\Delta} G}{G}-F\right) \geq\left[-\frac{4 b}{t} \Lambda^{2}-\frac{8 b}{t} n^{3} \Lambda E-M n+\lambda d\left(\frac{1}{t}+1\right)\right] I_{n},
$$

if we choose $d=d(n, \Lambda, \lambda, M, E)$ large enough, then

$$
2 D_{G}+A\left(\frac{\partial_{t} G-\tilde{\Delta} G}{G}-F\right) \geq\left(\frac{1}{t}+1\right) I_{n} .
$$

Estimate of $\partial_{t} F+F\left(\frac{\partial_{t} G-\tilde{\Delta} G}{G}-F\right)$.

By direct calculations we have

$$
\begin{aligned}
& \partial_{t} F+F\left(\frac{\partial_{t} G-\tilde{\Delta} G}{G}-F\right) \\
= & \frac{\left(d-2+2 b \partial_{i} a^{i j} x_{j}\right)\left(b|x|^{2}-4 b^{2} a^{i j} x_{i} x_{j}+K\right)}{t^{3}} \\
& +\frac{\left(d b|x|^{2}-4 d b^{2} a^{i j} x_{i} x_{j}-4 b^{2} \partial_{t} a^{i j} x_{i} x_{j}\right)+K d-\left(d-2 b a^{i i}\right)\left(d-1+2 b \partial_{i} a^{i j} x_{j}\right)}{t^{2}} \\
& -\frac{d\left(2 d-2 b a^{i i}+2 b \partial_{i} a^{i j} x_{j}\right)-2 b \partial_{t} a^{i i}}{t}-d^{2} \\
& +2 \gamma K t^{-K-2}\left(K+1-d-t d-2 b \partial_{i} a^{i j} x_{j}\right) .
\end{aligned}
$$


Noticing that

$$
\begin{gathered}
\left|\partial_{i} a^{i j} x_{j}\right| \leq n^{2} \frac{E}{|x|}|x|=n^{2} E \\
a^{i j} x_{i} x_{j} \leq \Lambda|x|^{2},\left|\partial_{t} a^{i j} x_{i} x_{j}\right| \leq M \sum_{i, j}\left|x_{i}\right|\left|x_{j}\right| \leq M n|x|^{2}
\end{gathered}
$$

then we have

$$
\begin{aligned}
& \partial_{t} F+F\left(\frac{\partial_{t} G-\tilde{\Delta} G}{G}-F\right) \\
\geq & \frac{(d-C)\left[\left(b-4 b^{2} \Lambda\right)|x|^{2}+K\right]}{t^{3}}+\frac{\left(d b-4 d b^{2} \Lambda-C\right)|x|^{2}+K d-(d+C)^{2}}{t^{2}} \\
& -\frac{d(2 d+C)+C}{t}-d^{2}+2 \gamma K t^{-K-2}(K+1-3 d-C) .
\end{aligned}
$$

Recall that $b=\frac{1}{8 \Lambda}$, and we choose $d$ large enough, then

$$
\begin{aligned}
& \partial_{t} F+F\left(\frac{\partial_{t} G-\tilde{\Delta} G}{G}-F\right) \\
\geq & \frac{d\left(\frac{b}{2}|x|^{2}+K\right)}{2 t^{3}}+\frac{(d b / 2-C)|x|^{2}+K d-2 d^{2}}{t^{2}}-\frac{3 d^{2}}{t}-d^{2}+2 \gamma K t^{-K-2}(K-4 d) \\
\geq & \frac{d\left(b|x|^{2}+K\right)}{4 t^{3}}+\frac{(d b / 2-C)|x|^{2}+K d-12 d^{2}}{t^{2}}+2 \gamma K t^{-K-2}(K-4 d),
\end{aligned}
$$

Since $K=12 d$, then

$$
\partial_{t} F+F\left(\frac{\partial_{t} G-\tilde{\Delta} G}{G}-F\right) \geq \frac{d b\left(|x|^{2}+1\right)}{4 t^{3}}
$$

Estimate of $\tilde{\triangle} F_{0}$.

In order to estimate $\tilde{\Delta} F_{0}$ and $\left|\nabla\left(F-F_{0}\right)\right|$, we need some estimates about $\left\{a_{\epsilon}^{i j}\right\}$ which we put in Appendix A.

In fact, $\left\{a_{\epsilon}^{i j}\right\}$ satisfy the following properties:

$$
\begin{aligned}
& \text { i) } \lambda|\xi|^{2} \leq a_{\epsilon}^{i j}(x, t) \xi_{i} \xi_{j} \leq \Lambda|\xi|^{2}, \forall \xi \in \mathbb{R}^{n} ; \\
& \text { ii) }\left|\nabla a_{\epsilon}^{i j}(x, t)\right| \leq M ;\left|\nabla a_{\epsilon}^{i j}(x, t)\right| \leq \frac{2 E}{|x|} \text { when }|x| \geq 1 ; \\
& \text { iii) }\left|a_{\epsilon}^{i j}(x, t)-a^{i j}(x, t)\right| \leq 2 \Lambda ;\left|a_{\epsilon}^{i j}(x, t)-a^{i j}(x, t)\right| \leq \frac{E}{|x|} \text { when }|x| \geq 1 ; \\
& \text { iv) }\left|\partial_{k l} a_{\epsilon}^{i j}(x, t)\right| \leq c(n) M ;\left|\partial_{k l} a_{\epsilon}^{i j}(x, t)\right| \leq \frac{c(n) E}{|x|} \text { when }|x| \geq 1 .
\end{aligned}
$$

Direct calculations show that

$$
\begin{aligned}
\tilde{\triangle} F_{0}= & \frac{b}{t^{2}} \tilde{\triangle}\left(|x|^{2}\right)-\frac{4 b^{2}}{t^{2}} \tilde{\triangle}\left(a_{\epsilon}^{i j} x_{i} x_{j}\right)+\frac{2 b}{t} \tilde{\triangle}\left(a_{\epsilon}^{i i}\right) \\
= & \frac{2 b}{t^{2}}\left(a^{i i}+\partial_{i} a^{i j} x_{j}\right)-\frac{4 b^{2}}{t^{2}}\left[\left(a^{k l} \partial_{k l} a_{\epsilon}^{i j}+\partial_{k} a^{k l} \partial_{l} a_{\epsilon}^{i j}\right) x_{i} x_{j}\right. \\
& \left.+2\left(\partial_{k} a^{k j} a_{\epsilon}^{i j}+2 a^{k j} \partial_{k} a_{\epsilon}^{i j}\right) x_{i}+2 a^{i j} a_{\epsilon}^{i j}\right]+\frac{2 b}{t}\left(a^{k l} \partial_{k l} a_{\epsilon}^{i i}+\partial_{k} a^{k l} \partial_{l} a_{\epsilon}^{i i}\right) .
\end{aligned}
$$


Now we estimate the terms in the right side of the above identity. By (35) we have

$$
\left|a_{\epsilon}^{i j}\right|,\left|\nabla a_{\epsilon}^{i j}\right| \text { and }\left|\nabla^{2} a_{\epsilon}^{i j}\right| \text { are all bounded, }
$$

then

$$
\begin{gathered}
\left|a^{i i}+\partial_{i} a^{i j} x_{j}\right| \leq C(1+|x|) ; \\
\left|\left(a^{k l} \partial_{k l} a_{\epsilon}^{i j}+\partial_{k} a^{k l} \partial_{l} a_{\epsilon}^{i j}\right) x_{i} x_{j}\right| \leq C|x|^{2} \\
\left|\left(\partial_{k} a^{k j} a_{\epsilon}^{i j}+2 a^{k j} \partial_{k} a_{\epsilon}^{i j}\right) x_{i}\right| \leq C|x| ; \\
\left|a^{k l} \partial_{k l} a_{\epsilon}^{i i}+\partial_{k} a^{k l} \partial_{l} a_{\epsilon}^{i i}\right| \leq C .
\end{gathered}
$$

Thus

$$
\tilde{\triangle} F_{0} \geq-\frac{C(|x|+1)}{t^{2}}-\frac{C\left(|x|^{2}+|x|+1\right)}{t^{2}}-\frac{C}{t} \geq-\frac{C\left(|x|^{2}+1\right)}{t^{3}} .
$$

Estimate of $\left|\nabla\left(F-F_{0}\right)\right|$.

Since

$$
F-F_{0}=\frac{4 b^{2}\left(a_{\epsilon}^{i j}-a^{i j}\right) x_{i} x_{j}}{t^{2}}-\frac{2 b\left(a_{\epsilon}^{i i}-a^{i i}\right)}{t},
$$

then

$$
\begin{aligned}
\left|\nabla\left(F-F_{0}\right)\right| & =\left|\frac{4 b^{2}}{t^{2}}\left(\nabla a_{\epsilon}^{i j}-\nabla a^{i j}\right) x_{i} x_{j}+\frac{8 b^{2}}{t^{2}}\left(a_{\epsilon}^{i j}-a^{i j}\right) x_{i} \nabla x_{j}-\frac{2 b}{t}\left(\nabla a_{\epsilon}^{i i}-\nabla a^{i i}\right)\right| \\
& \leq \frac{4 b^{2}}{t^{2}}\left|\nabla a_{\epsilon}^{i j}-\nabla a^{i j}\right|\left|x_{i}\right|\left|x_{j}\right|+\frac{8 b^{2}}{t^{2}} \sum_{j}\left|a_{\epsilon}^{i j}-a^{i j}\right|\left|x_{i}\right|+\frac{2 b}{t}\left|\nabla a_{\epsilon}^{i i}-\nabla a^{i i}\right| .
\end{aligned}
$$

We now estimate the terms in the right side of the above inequality. By ii) of (35), when $|x|<1$,

$$
\left|\nabla a_{\epsilon}^{i j}-\nabla a^{i j}\right|\left|x_{i}\right|\left|x_{j}\right| \leq 2 M \sum_{i, j}\left|x_{i}\right|\left|x_{j}\right| \leq 2 M n|x|^{2} \leq 2 M n,
$$

and when $|x| \geq 1$,

$$
\left|\nabla a_{\epsilon}^{i j}-\nabla a^{i j}\right|\left|x_{i}\right|\left|x_{j}\right| \leq\left(\frac{2 E}{|x|}+\frac{E}{|x|}\right) \sum_{i, j}\left|x_{i}\right|\left|x_{j}\right| \leq \frac{3 E}{|x|} n|x|^{2}=3 n E|x| .
$$

Then we have

$$
\left|\nabla a_{\epsilon}^{i j}-\nabla a^{i j}\right|\left|x_{i}\right|\left|x_{j}\right| \leq C(|x|+1) .
$$

By $i$ ) and $i i i)$ of (35) we have

$$
\begin{gathered}
\sum_{j}\left|a_{\epsilon}^{i j}-a^{i j}\right|\left|x_{i}\right| \leq 2 n^{2} \Lambda|x|, \\
\left|\nabla a_{\epsilon}^{i i}-\nabla a^{i i}\right| \leq 2 M n .
\end{gathered}
$$

With the above three estimates we have

$$
\left|\nabla\left(F-F_{0}\right)\right| \leq \frac{C(|x|+1)}{t^{2}}+\frac{C|x|}{t^{2}}+\frac{C}{t} \leq \frac{C(|x|+1)}{t^{2}} .
$$

Thus we proved Lemma 3.3. 
3.2. Proof of Proposition 1.4. Before we prove proposition 1.4, we need to prove a result as another version of Corollary 3.2 .

In (26) , we let $\Phi=\gamma\left(t^{-K}-1\right) x_{n}^{\alpha}-\frac{b \psi+K}{2 t}, G=e^{2 \Phi}, v=e^{\Phi} u$ and we denote

$$
B=2 D_{G}+A\left(\frac{\partial_{t} G-\tilde{\Delta} G}{G}-F\right) .
$$

Then the third term of the left hand side of (26) is

$$
\begin{aligned}
& -\int_{Q} u\left\langle A \nabla\left(F-F_{0}\right), \nabla u\right\rangle e^{2 \Phi} d x d t \\
= & -\int_{Q} v\left\langle A \nabla\left(F-F_{0}\right), \nabla v-\nabla \Phi v\right\rangle d x d t \\
= & -\int_{Q} v\left\langle A \nabla\left(F-F_{0}\right), \nabla v\right\rangle d x d t+\int_{Q}\left\langle A \nabla\left(F-F_{0}\right), \nabla \Phi\right\rangle v^{2} d x d t .
\end{aligned}
$$

We use the above identity and rewrite (26) as

$$
\begin{aligned}
\frac{1}{2} \int_{Q} M_{1} v^{2} d x d t+ & \int_{Q}\langle B \nabla u, \nabla u\rangle e^{2 \Phi} d x d t-\int_{Q} v\left\langle A \nabla\left(F-F_{0}\right), \nabla v\right\rangle d x d t \\
& =2 \int_{Q} L u(P u-L u) e^{2 \Phi} d x d t
\end{aligned}
$$

where

$$
\begin{gathered}
M_{1}=\partial_{t} F+F\left(\frac{\partial_{t} G-\tilde{\Delta} G}{G}-F\right)+\tilde{\Delta} F_{0} \\
B=4 A D^{2} \Phi A+2 \partial_{l} \Phi\left(a^{k i} \partial_{k} a^{l j}+a^{k j} \partial_{k} a^{l i}-a^{k l} \partial_{k} a^{i j}\right)+\partial_{t} a^{i j}+A\left(\frac{\partial_{t} G-\tilde{\Delta} G}{G}-F\right) .
\end{gathered}
$$

We rewrite $\Phi$ as the following:

$$
\begin{aligned}
& \Phi=\Phi_{1}+\Phi_{2}, \\
& \Phi_{1}=\gamma f(t) x_{n}^{\alpha}, \quad f(t)=t^{-K}-1, \\
& \Phi_{2}=-\frac{b \psi+K}{2 t} .
\end{aligned}
$$

The function $\psi$ has the following properties which we will prove in Appendix B:

$$
\begin{aligned}
& \text { i) } \psi \geq \frac{|x|^{2}}{2} \\
& \text { ii) } D^{2} \psi \leq C\left(\frac{\Lambda}{\lambda}\right) I_{n} ; \\
& \text { iii) }|\nabla \psi| \leq 4\left(\frac{\Lambda}{\lambda}+1\right)^{2}|x|,\left|\nabla^{k} \psi\right| \leq \frac{C\left(n, \frac{\Lambda}{\lambda}\right)}{|x|^{k-2}}, \quad k=2,3,4 ; \\
& \text { iv) } a^{n i} \partial_{i} \psi \leq C(\Lambda, \lambda) x_{n} .
\end{aligned}
$$

By direct calculations we have

$$
\frac{\partial_{t} G-\tilde{\Delta} G}{G}=2 \partial_{t} \Phi-2 a^{i j} \partial_{i j} \Phi-2 \partial_{i} a^{i j} \partial_{j} \Phi-4\langle A \nabla \Phi, \nabla \Phi\rangle .
$$


BACKWARD UNIQUENESS FOR PARABOLIC OPERATORSWITH VARIABLE COEFFICIENTS IN A HALF SPACE

Let

$$
F=2 \partial_{t} \Phi-2 a^{i j} \partial_{i j} \Phi-4\langle A \nabla \Phi, \nabla \Phi\rangle-H
$$

where $H$ is a positive smooth function to be determined. Let

$$
F_{0}=2 \partial_{t} \Phi-2 a_{\epsilon}^{i j} \partial_{i j} \Phi-4 a_{\epsilon}^{i j} \partial_{i} \Phi \partial_{j} \Phi-H
$$

We estimate matrix $B$ first. Direct calculations show that

$$
\begin{aligned}
B & =4 A D^{2} \Phi A+2 \partial_{l} \Phi\left(a^{k i} \partial_{k} a^{l j}+a^{k j} \partial_{k} a^{l i}-a^{k l} \partial_{k} a^{i j}\right)+\partial_{t} a^{i j}+A\left(-2 \partial_{k} a^{k l} \partial_{l} \Phi+H\right) \\
& =4 A D^{2} \Phi A+2 \partial_{l} \Phi\left(a^{k i} \partial_{k} a^{l j}+a^{k j} \partial_{k} a^{l i}-a^{k l} \partial_{k} a^{i j}-a^{i j} \partial_{k} a^{k l}\right)+\partial_{t} a^{i j}+H A .
\end{aligned}
$$

We estimate the lower bounds of the matrices in the right side of the above identity. First, by $i i)$ of (38) we have

$$
A D^{2} \Phi A=A D^{2} \Phi_{1} A-\frac{b}{2 t} A D^{2} \psi A \geq \partial_{n n} \Phi_{1} a^{i n} a^{n j}-\frac{C}{t} I_{n} .
$$

Second, we estimate matrix $\partial_{l} \Phi a^{k i} \partial_{k} a^{l j}$ and $\partial_{t} a^{i j}$.

For any $\xi \in \mathbb{R}^{n}$,

$$
\begin{aligned}
\left|\partial_{l} \Phi a^{k i} \partial_{k} a^{l j} \xi_{i} \xi_{j}\right| & \leq n \Lambda \frac{E}{|x|} \sum_{l, i, j}\left|\partial_{l} \Phi\right| \xi_{i}|| \xi_{j} \mid \\
& \leq \frac{n^{2} \Lambda E}{|x|}|\xi|^{2} \sum_{l}\left|\partial_{l} \Phi\right| \\
& \leq \frac{n^{2} \Lambda E}{|x|}\left(\partial_{n} \Phi_{1}+\frac{C}{t}|\nabla \psi|\right)|\xi|^{2},
\end{aligned}
$$

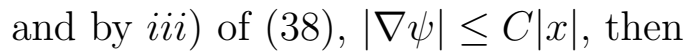

$$
\left|\partial_{l} \Phi a^{k i} \partial_{k} a^{l j} \xi_{i} \xi_{j}\right| \leq\left(n^{2} \Lambda E \frac{\partial_{n} \Phi_{1}}{|x|}+\frac{C}{t}\right)|\xi|^{2},
$$

thus

again,

$$
-\left(n^{2} \Lambda E \frac{\partial_{n} \Phi_{1}}{|x|}+\frac{C}{t}\right) I_{n} \leq \partial_{l} \Phi a^{k i} \partial_{k} a^{l j} \leq\left(n^{2} \Lambda E \frac{\partial_{n} \Phi_{1}}{|x|}+\frac{C}{t}\right) I_{n} ;
$$

$$
\left|\partial_{t} a^{i j} \xi_{i} \xi_{j}\right| \leq M \sum_{i, j}\left|\xi_{i}\right|\left|\xi_{j}\right| \leq M n|\xi|^{2}
$$

then

$$
\partial_{t} a^{i j} \geq-M n I_{n}
$$

Consequently,

$$
\begin{aligned}
B & \geq 4 \partial_{n n} \Phi_{1} a^{i n} a^{n j}-\frac{C}{t} I_{n}-8\left(n^{2} \Lambda E \frac{\partial_{n} \Phi_{1}}{|x|}+\frac{C}{t}\right) I_{n}-M n I_{n}+H A \\
& \geq 4 \partial_{n n} \Phi_{1} a^{i n} a^{n j}-\left(8 n^{2} \Lambda E \frac{\partial_{n} \Phi_{1}}{|x|}+\frac{C}{t}\right) I_{n}+H A+\frac{1}{t} I_{n} \\
& \equiv \tilde{B}+\frac{1}{t} I_{n},
\end{aligned}
$$

where

$$
\tilde{B}=4 \partial_{n n} \Phi_{1} a^{i n} a^{n j}-\left(8 n^{2} \Lambda E \frac{\partial_{n} \Phi_{1}}{|x|}+\frac{C}{t}\right) I_{n}+H A
$$


To make $\tilde{B}$ positive, we choose

$$
H=16 n^{2} \frac{\Lambda}{\lambda} E \frac{\partial_{n} \Phi_{1}}{|x|}+\frac{d}{t}
$$

where $d$ is a positive constant to be determined.

Since $\tilde{B}$ is differentiable, then by (40) we have

$$
\begin{aligned}
& \int_{Q}\langle B \nabla u, \nabla u\rangle e^{2 \Phi} d x d t \\
\geq & \int_{Q}\langle\tilde{B} \nabla u, \nabla u\rangle e^{2 \Phi} d x d t+\int_{Q} e^{2 \Phi} \frac{|\nabla u|^{2}}{t} d x d t \\
= & \int_{Q}\langle\tilde{B} \nabla v, \nabla v\rangle d x d t+\int_{Q}[\langle\tilde{B} \nabla \Phi, \nabla \Phi\rangle+\operatorname{div}(\tilde{B} \nabla \Phi)] v^{2} d x d t+\int_{Q} e^{2 \Phi} \frac{|\nabla u|^{2}}{t} d x d t .
\end{aligned}
$$

By (37), (43) and the Cauchy inequality, we have

$$
\begin{aligned}
& \int_{Q} e^{2 \Phi} \frac{|\nabla u|^{2}}{t} d x d t+\int_{Q}\langle\tilde{B} \nabla v, \nabla v\rangle d x d t+\int_{Q} M_{2} v^{2} d x d t \\
& -\int_{Q} v\left\langle A \nabla\left(F-F_{0}\right), \nabla v\right\rangle d x d t \leq \int_{Q} e^{2 \Phi}|P u|^{2} d x d t,
\end{aligned}
$$

where

$$
M_{2}=\langle\tilde{B} \nabla \Phi, \nabla \Phi\rangle+\operatorname{div}(\tilde{B} \nabla \Phi)+\frac{1}{2} \partial_{t} F+\frac{1}{2} F\left(\frac{\partial_{t} G-\tilde{\Delta} G}{G}-F\right)+\frac{1}{2} \tilde{\Delta} F_{0} .
$$

We use inequality (44) to prove Proposition 1.4. We also need some estimates which we list in the following lemma. We will prove this lemma later.

Lemma 3.4. Set $b=\frac{1}{64 \Lambda\left(\frac{\Lambda}{\lambda}+1\right)^{4}}, E_{0}=\frac{\lambda}{16 n^{2} \frac{\Lambda}{\lambda}\left(\frac{\Lambda}{\lambda}+1\right)}, \alpha=1+\frac{E}{E_{0}}$ and $K=13 \frac{\Lambda}{\lambda} d$. We take $d=d(n, \Lambda, \lambda, M, E)$ large enough, when $E<E_{0}$, we have

$$
\begin{gathered}
\tilde{B} \geq 8 n^{2} \Lambda E\left(\frac{\partial_{n} \Phi_{1}}{|x|}+\frac{1}{t}\right) I_{n} ; \\
M_{2} \geq 2\left[(\alpha-1) \lambda^{2}-\left(16 n^{2} \frac{\Lambda}{\lambda}+8 n^{2}+4 n\right) \Lambda E\right] \frac{\left(\partial_{n} \Phi_{1}\right)^{3}}{|x|}+\frac{b d|x|^{2}}{16 t^{3}}+\frac{1}{t^{3}} ; \\
\left|\nabla\left(F-F_{0}\right)\right| \leq 32 n E\left[\frac{\left(\partial_{n} \Phi_{1}\right)^{2}}{|x|}+\frac{C|x|}{t^{2}}\right] .
\end{gathered}
$$

Then by applying (45) in Lemma 3.4, we have

$$
\int_{Q}\langle\tilde{B} \nabla v, \nabla v\rangle d x d t \geq 8 n^{2} \Lambda E \int_{Q}\left(\frac{\partial_{n} \Phi_{1}}{|x|}+\frac{1}{t}\right)|\nabla v|^{2} d x d t
$$

By (46) we have

$$
\begin{aligned}
\int_{Q} M_{2} v^{2} d x d t \geq & 2 \int_{Q}\left[(\alpha-1) \lambda^{2}-\left(16 n^{2} \frac{\Lambda}{\lambda}+8 n^{2}+4 n\right) \Lambda E\right] \frac{\left(\partial_{n} \Phi_{1}\right)^{3}}{|x|} v^{2} d x d t \\
& +\int_{Q}\left(\frac{b d|x|^{2}}{16 t^{3}}+\frac{1}{t^{3}}\right) v^{2} d x d t .
\end{aligned}
$$


BACKWARD UNIQUENESS FOR PARABOLIC OPERATORSWITH VARIABLE COEFFICIENTS IN A HALF SPAGI By (47)

$$
\begin{aligned}
\left|\int_{Q} v\left\langle A \nabla\left(F-F_{0}\right), \nabla v\right\rangle d x d t\right| & \leq \Lambda \int_{Q}\left|\nabla\left(F-F_{0}\right)\right||v||\nabla v| d x d t \\
& \leq 32 n \Lambda E \int_{Q}\left[\frac{\left(\partial_{n} \Phi_{1}\right)^{2}}{|x|}+\frac{C|x|}{t^{2}}\right]|v||\nabla v| d x d t
\end{aligned}
$$

Using the Cauchy inequality, we have

$$
\begin{aligned}
\left|\int_{Q} v\left\langle A \nabla\left(F-F_{0}\right), \nabla v\right\rangle d x d t\right| \leq & 32 \Lambda E \int_{Q}\left[\frac{\left(\partial_{n} \Phi_{1}\right)^{3}}{|x|}+\frac{C|x|^{2}}{t^{3}}\right] v^{2} d x d t \\
& +8 n^{2} \Lambda E \int_{Q}\left(\frac{\partial_{n} \Phi_{1}}{|x|}+\frac{1}{t}\right)|\nabla v|^{2} d x d t .
\end{aligned}
$$

Because of (44), (48), (49) and (50), we have

$$
\begin{aligned}
\int_{Q} e^{2 \Phi}|P u|^{2} d x d t \geq & \int_{Q} 2\left[(\alpha-1) \lambda^{2}-\left(16 n^{2} \frac{\Lambda}{\lambda}+8 n^{2}+4 n+16\right) \Lambda E\right] \frac{\left(\partial_{n} \Phi_{1}\right)^{3}}{|x|} v^{2} d x d t \\
& +\int_{Q}\left[\frac{(b d-C)|x|^{2}}{16 t^{3}}+\frac{1}{t^{3}}\right] v^{2} d x d t+\int_{Q} e^{2 \Phi} \frac{|\nabla u|^{2}}{t} d x d t,
\end{aligned}
$$

Since

$$
16 n^{2} \frac{\Lambda}{\lambda}+8 n^{2}+4 n+16 \leq 16 n^{2}\left(\frac{\Lambda}{\lambda}+1\right),
$$

and if we take $d$ large enough, then

$$
\begin{aligned}
\int_{Q} e^{2 \Phi}|P u|^{2} d x d t \geq & \int_{Q} 2\left[(\alpha-1) \lambda^{2}-16 n^{2}\left(\frac{\Lambda}{\lambda}+1\right) \Lambda E\right] \frac{\left(\partial_{n} \Phi_{1}\right)^{3}}{|x|} v^{2} d x d t \\
& +\int_{Q} e^{2 \Phi}\left(\frac{|u|^{2}}{t^{3}}+\frac{|\nabla u|^{2}}{t}\right) d x d t .
\end{aligned}
$$

Notice that

thus

$$
(\alpha-1) \lambda^{2}-16 n^{2}\left(\frac{\Lambda}{\lambda}+1\right) \Lambda E=0
$$

$$
\int_{Q} e^{2 \Phi}|P u|^{2} d x d t \geq \int_{Q} e^{2 \Phi}\left(\frac{|u|^{2}}{t^{3}}+\frac{|\nabla u|^{2}}{t}\right) d x d t .
$$

Thus we proved Carleman inequality (13).

Proof of Lemma 3.4 .

Estimate of $\tilde{B}$.

By (41) and (42) we have

$$
\tilde{B} \geq\left[-\left(8 n^{2} \Lambda E \frac{\partial_{n} \Phi_{1}}{|x|}+\frac{C}{t}\right)+\lambda H\right] I_{n}=\left(8 n^{2} \Lambda E \frac{\partial_{n} \Phi_{1}}{|x|}+\frac{\lambda d-C}{t}\right) I_{n},
$$

if we take $d=d(n, \Lambda, \lambda, M, E)$ large enough, then

$$
\tilde{B} \geq 8 n^{2} \Lambda E\left(\frac{\partial_{n} \Phi_{1}}{|x|}+\frac{1}{t}\right) I_{n}
$$


Estimate of $M_{2}$.

In order to estimate $M_{2}$, we have to divide $M_{2}$ into several parts and estimate each of them:

$$
M_{2}=J_{1}+J_{2}+J_{3}+J_{4}+J_{5}+J_{6}
$$

where

$$
\begin{aligned}
J_{1}= & 4 \partial_{n n} \Phi_{1}\left(a^{n i} \partial_{i} \Phi\right)^{2} ; \\
J_{2}= & -\left(8 n^{2} \Lambda E \frac{\partial_{n} \Phi_{1}}{|x|}+\frac{C}{t}\right)|\nabla \Phi|^{2}-\left(H-4 \partial_{i} a^{i j} \partial_{j} \Phi\right)\langle A \nabla \Phi, \nabla \Phi\rangle \\
& +\langle A \nabla H, \nabla \Phi\rangle-8 n^{2} \Lambda E\left\langle\nabla\left(\frac{\partial_{n} \Phi_{1}}{|x|}\right), \nabla \Phi\right\rangle-\left(8 n^{2} \Lambda E \frac{\partial_{n} \Phi_{1}}{|x|}+\frac{C}{t}\right) \Delta \Phi ; \\
J_{3}= & 4 \partial_{n n} \Phi_{1} \partial_{i}\left(a^{i n} a^{n j}\right) \partial_{j} \Phi+4 \partial_{n}^{3} \Phi_{1} a^{n n} a^{n j} \partial_{j} \Phi+4 \partial_{n n} \Phi_{1} a^{i n} a^{n j} \partial_{i j} \Phi ; \\
J_{4}= & \partial_{t t} \Phi+\partial_{t} \Phi\left(H-2 \partial_{i} a^{i j} \partial_{j} \Phi\right)-\partial_{t} a^{i j} \partial_{i j} \Phi-a^{i j} \partial_{i j t} \Phi \\
& +2 \partial_{i} a^{i j} \partial_{j} \Phi\left(H+a^{i j} \partial_{i j} \Phi\right)-\frac{1}{2} \partial_{t} H-\frac{1}{2} H^{2} ; \\
J_{5}= & -2 \partial_{t}\langle A \nabla \Phi, \nabla \Phi\rangle \\
J_{6}= & \frac{1}{2} \tilde{\Delta} F_{0} .
\end{aligned}
$$

Estimate of $J_{1}$.

$$
J_{1}=4 \partial_{n n} \Phi_{1}\left(a^{n n} \partial_{n} \Phi_{1}+a^{n i} \partial_{i} \Phi_{2}\right)^{2},
$$

By the Cauchy inequality $(a+b)^{2} \geq \delta a^{2}-\frac{\delta}{1-\delta} b^{2}, 0<\delta<1$, we have

$$
\begin{aligned}
J_{1} & \geq 4 \partial_{n n} \Phi_{1}\left[\frac{x_{n}}{2|x|}\left(a^{n n} \partial_{n} \Phi_{1}\right)^{2}-\frac{x_{n}}{2|x|-x_{n}}\left(a^{n i} \partial_{i} \Phi_{2}\right)^{2}\right] \\
& \geq 4 \partial_{n n} \Phi_{1}\left[\frac{x_{n}}{2|x|} \lambda^{2}\left(\partial_{n} \Phi_{1}\right)^{2}-\frac{x_{n}}{|x|}\left(a^{n i} \partial_{i} \Phi_{2}\right)^{2}\right] \\
& =2(\alpha-1) \frac{\partial_{n} \Phi_{1}}{|x|}\left[\lambda^{2}\left(\partial_{n} \Phi_{1}\right)^{2}-2\left(a^{n i} \partial_{i} \Phi_{2}\right)^{2}\right]
\end{aligned}
$$

Again by the Cauchy inequality we have

$$
\left(a^{n i} \partial_{i} \Phi_{2}\right)^{2} \leq \sum_{i}\left(a^{n i}\right)^{2}\left|\nabla \Phi_{2}\right|^{2} \leq \frac{\Lambda^{2} b^{2}}{4 t^{2}}|\nabla \psi|^{2},
$$

then

$$
\begin{aligned}
J_{1} & \geq 2(\alpha-1) \frac{\partial_{n} \Phi_{1}}{|x|}\left[\lambda^{2}\left(\partial_{n} \Phi_{1}\right)^{2}-\frac{\Lambda^{2} b^{2}}{2 t^{2}}|\nabla \psi|^{2}\right] \\
& =2(\alpha-1) \lambda^{2} \frac{\left(\partial_{n} \Phi_{1}\right)^{3}}{|x|}-\frac{C}{t^{2}} \frac{\partial_{n} \Phi_{1}}{|x|}|\nabla \psi|^{2} .
\end{aligned}
$$

By $i i i)$ of (38), $|\nabla \psi| \leq C|x|$, then

$$
J_{1} \geq 2(\alpha-1) \lambda^{2} \frac{\left(\partial_{n} \Phi_{1}\right)^{3}}{|x|}-\frac{C}{t^{2}} \partial_{n} \Phi_{1}|x| .
$$


BACKWARD UNIQUENESS FOR PARABOLIC OPERATORSWITH VARIABLE COEFFICIENTS IN A HALF SPAC2E Using the Cauchy inequality, we obtain

$$
J_{1} \geq 2(\alpha-1) \lambda^{2} \frac{\left(\partial_{n} \Phi_{1}\right)^{3}}{|x|}-\frac{C}{t}\left(\partial_{n} \Phi_{1}\right)^{2}-\frac{C}{t^{3}}|x|^{2} .
$$

Estimate of $J_{2}$.

Let's estimate $\partial_{i} a^{i j} \partial_{j} \Phi$ first. We will use this estimate afterwards again.

$$
\left|\partial_{i} a^{i j} \partial_{j} \Phi\right| \leq \frac{n E}{|x|} \sum_{j}\left|\partial_{j} \Phi\right| \leq \frac{n E}{|x|}\left(\partial_{n} \Phi_{1}+\frac{C}{t}|\nabla \psi|\right) .
$$

Recall that $|\nabla \psi| \leq C|x|$, then

$$
\left|\partial_{i} a^{i j} \partial_{j} \Phi\right| \leq n E \frac{\partial_{n} \Phi_{1}}{|x|}+\frac{C}{t} .
$$

By (51) we have

$$
\begin{aligned}
J_{2} \geq & -\left(8 n^{2} \Lambda E \frac{\partial_{n} \Phi_{1}}{|x|}+\frac{C}{t}\right)|\nabla \Phi|^{2}-\left[\left(16 n^{2} \frac{\Lambda}{\lambda}+4 n\right) E \frac{\partial_{n} \Phi_{1}}{|x|}+\frac{d+C}{t}\right] \Lambda|\nabla \Phi|^{2} \\
& -\Lambda|\nabla H||\nabla \Phi|-8 n^{2} \Lambda E\left|\nabla\left(\frac{\partial_{n} \Phi_{1}}{|x|}\right)\right||\nabla \Phi|-\left(8 n^{2} \Lambda E \frac{\partial_{n} \Phi_{1}}{|x|}+\frac{C}{t}\right) \Delta \Phi \\
= & -\left[\left(16 n^{2} \frac{\Lambda}{\lambda}+8 n^{2}+4 n\right) \Lambda E \frac{\partial_{n} \Phi_{1}}{|x|}+\frac{d \Lambda+C}{t}\right]|\nabla \Phi|^{2} \\
& -\left(16 n^{2} \frac{\Lambda}{\lambda}+8 n^{2}\right) \Lambda E\left|\nabla\left(\frac{\partial_{n} \Phi_{1}}{|x|}\right)\right||\nabla \Phi|-\left(8 n^{2} \Lambda E \frac{\partial_{n} \Phi_{1}}{|x|}+\frac{C}{t}\right) \Delta \Phi .
\end{aligned}
$$

Because

$$
\left|\nabla\left(\frac{\partial_{n} \Phi_{1}}{|x|}\right)\right| \leq \frac{\partial_{n} \Phi_{1}}{x_{n}|x|} \leq \partial_{n} \Phi_{1}
$$

and we have by $i i i)$ of (38) that

$$
\Delta \Phi=\partial_{n n} \Phi_{1}-\frac{b}{2 t} \Delta \psi \leq \partial_{n n} \Phi_{1}+\frac{C}{t}
$$

then

$$
\begin{aligned}
J_{2} \geq & -\left[\left(16 n^{2} \frac{\Lambda}{\lambda}+8 n^{2}+4 n\right) \Lambda E \frac{\partial_{n} \Phi_{1}}{|x|}+\frac{d \Lambda+C}{t}\right]|\nabla \Phi|^{2}-C \partial_{n} \Phi_{1}|\nabla \Phi| \\
& -\left(8 n^{2} \Lambda E \frac{\partial_{n} \Phi_{1}}{|x|}+\frac{C}{t}\right)\left(\partial_{n n} \Phi_{1}+\frac{C}{t}\right) \\
\geq & -\left[\left(16 n^{2} \frac{\Lambda}{\lambda}+8 n^{2}+4 n\right) \Lambda E \frac{\partial_{n} \Phi_{1}}{|x|}+\frac{d \Lambda+C}{t}\right]|\nabla \Phi|^{2}-C \partial_{n} \Phi_{1}|\nabla \Phi| \\
& -C\left(\partial_{n} \Phi_{1}\right)^{2}-\frac{C}{t} \partial_{n} \Phi_{1}-\frac{C}{t^{2}} .
\end{aligned}
$$

By the Cauchy inequality we have

$$
C \partial_{n} \Phi_{1}|\nabla \Phi| \leq C\left(\partial_{n} \Phi_{1}\right)^{2}+C|\nabla \Phi|^{2}, \frac{C}{t} \partial_{n} \Phi_{1} \leq C\left(\partial_{n} \Phi_{1}\right)^{2}+\frac{C}{t^{2}},
$$


then

$$
\begin{aligned}
J_{2} \geq & -\left[\left(16 n^{2} \frac{\Lambda}{\lambda}+8 n^{2}+4 n\right) \Lambda E \frac{\partial_{n} \Phi_{1}}{|x|}+\frac{d \Lambda+C}{t}\right]|\nabla \Phi|^{2}-C\left(\partial_{n} \Phi_{1}\right)^{2}-\frac{C}{t^{2}} \\
\geq & -2\left[\left(16 n^{2} \frac{\Lambda}{\lambda}+8 n^{2}+4 n\right) \Lambda E \frac{\partial_{n} \Phi_{1}}{|x|}+\frac{d \Lambda+C}{t}\right]\left[\left(\partial_{n} \Phi_{1}\right)^{2}+\frac{b^{2}}{4 t^{2}}|\nabla \psi|^{2}\right]-C\left(\partial_{n} \Phi_{1}\right)^{2}-\frac{C}{t^{2}} \\
\geq & -2\left(16 n^{2} \frac{\Lambda}{\lambda}+8 n^{2}+4 n\right) \Lambda E \frac{\left(\partial_{n} \Phi_{1}\right)^{3}}{|x|}-\frac{2 d \Lambda+C}{t}\left(\partial_{n} \Phi_{1}\right)^{2}-\frac{C}{t^{2}} \frac{\partial_{n} \Phi_{1}}{|x|}|\nabla \psi|^{2} \\
& -\frac{(d \Lambda+C) b^{2}}{2 t^{3}}|\nabla \psi|^{2}-\frac{C}{t^{2}} .
\end{aligned}
$$

Taking in account that $|\nabla \psi| \leq C|x|$ and using the Cauchy inequality, we have that

$$
\frac{C}{t^{2}} \frac{\partial_{n} \Phi_{1}}{|x|}|\nabla \psi|^{2} \leq \frac{C}{t^{2}} \partial_{n} \Phi_{1}|x| \leq \frac{C}{t}\left(\partial_{n} \Phi_{1}\right)^{2}+\frac{C}{t^{3}}|x|^{2}
$$

then

$$
\begin{aligned}
J_{2} \geq & -2\left(16 n^{2} \frac{\Lambda}{\lambda}+8 n^{2}+4 n\right) \Lambda E \frac{\left(\partial_{n} \Phi_{1}\right)^{3}}{|x|}-\frac{2 d \Lambda+C}{t}\left(\partial_{n} \Phi_{1}\right)^{2}-\frac{C}{t^{3}}|x|^{2} \\
& -\frac{d b^{2} \Lambda+C}{2 t^{3}}|\nabla \psi|^{2}-\frac{C}{t^{2}} .
\end{aligned}
$$

In the following, we always use the fact that

$$
\left|\nabla^{k} \psi\right| \leq \frac{C}{|x|^{k-2}}, \quad k=1,2,3,4 .
$$

Estimate of $J_{3}$.

$$
J_{3}=4 \partial_{n n} \Phi_{1}\left(\partial_{i} a^{i n} a^{n j}+a^{i n} \partial_{i} a^{n j}\right) \partial_{j} \Phi+4 \partial_{n}^{3} \Phi_{1} a^{n n} a^{n j} \partial_{j} \Phi+4 \partial_{n n} \Phi_{1} a^{i n} a^{n j} \partial_{i j} \Phi .
$$

Next we estimate the terms of $J_{3}$.

$$
\begin{gathered}
\left|\left(\partial_{i} a^{i n} a^{n j}+a^{i n} \partial_{i} a^{n j}\right) \partial_{j} \Phi\right| \leq C|\nabla \Phi| \leq C\left(\partial_{n} \Phi_{1}+\frac{|\nabla \psi|}{t}\right) \leq C\left(\partial_{n} \Phi_{1}+\frac{|x|}{t}\right) \\
\left|a^{n n} a^{n j} \partial_{j} \Phi\right| \leq C|\nabla \Phi| \leq C\left(\partial_{n} \Phi_{1}+\frac{|\nabla \psi|}{t}\right) \leq C\left(\partial_{n} \Phi_{1}+\frac{|x|}{t}\right) \\
\left|a^{i n} a^{n j} \partial_{i j} \Phi\right| \leq C\left|\nabla^{2} \Phi\right| \leq C\left(\partial_{n n} \Phi_{1}+\frac{\left|\nabla^{2} \psi\right|}{t}\right) \leq C\left(\partial_{n n} \Phi_{1}+\frac{1}{t}\right) .
\end{gathered}
$$

Combining the above estimates, we have

$$
\begin{aligned}
J_{3} & \geq-C \partial_{n n} \Phi_{1}\left(\partial_{n} \Phi_{1}+\frac{|x|}{t}\right)-C\left|\partial_{n}^{3} \Phi_{1}\right|\left(\partial_{n} \Phi_{1}+\frac{|x|}{t}\right)-C \partial_{n n} \Phi_{1}\left(\partial_{n n} \Phi_{1}+\frac{1}{t}\right) \\
& \geq-C\left[\left(\partial_{n} \Phi_{1}\right)^{2}+\frac{1}{t} \partial_{n} \Phi_{1}+\partial_{n} \Phi_{1} \frac{|x|}{t}\right] .
\end{aligned}
$$

Using the Cauchy inequality, we have

$$
J_{3} \geq-C\left[\left(\partial_{n} \Phi_{1}\right)^{2}+\frac{1}{t^{2}}+\frac{|x|^{2}}{t^{2}}\right] .
$$


BACKWARD UNIQUENESS FOR PARABOLIC OPERATORSWITH VARIABLE COEFFICIENTS IN A HALF SPACB

Estimate of $J_{4}$.

$$
\begin{aligned}
J_{4}= & \partial_{t t} \Phi+\partial_{t} \Phi\left(H-2 \partial_{i} a^{i j} \partial_{j} \Phi\right)-\partial_{t} a^{i j} \partial_{i j} \Phi-a^{i j} \partial_{i j t} \Phi \\
& +2 \partial_{i} a^{i j} \partial_{j} \Phi\left(H+a^{i j} \partial_{i j} \Phi\right)-\frac{1}{2} \partial_{t} H-\frac{1}{2} H^{2} .
\end{aligned}
$$

We estimate the terms of $J_{4}$.

In fact

$$
\begin{gathered}
\partial_{t t} \Phi=\partial_{t t} \Phi_{1}+\partial_{t t} \Phi_{2}=\partial_{t t} \Phi_{1}-\frac{b \psi+K}{t^{3}} \\
\partial_{t} \Phi\left(H-2 \partial_{i} a^{i j} \partial_{j} \Phi\right)=\partial_{t} \Phi_{1}\left(H-2 \partial_{i} a^{i j} \partial_{j} \Phi\right)+\frac{b \psi+K}{2 t^{2}}\left(H-2 \partial_{i} a^{i j} \partial_{j} \Phi\right) .
\end{gathered}
$$

Recall (53)

$$
\left|\partial_{i} a^{i j} \partial_{j} \Phi\right| \leq n E \frac{\partial_{n} \Phi_{1}}{|x|}+\frac{C}{t}
$$

and notice that $\partial_{t} \Phi_{1}<0$, then we have

$$
\begin{aligned}
& \partial_{t} \Phi\left(H-2 \partial_{i} a^{i j} \partial_{j} \Phi\right) \\
\geq & \partial_{t} \Phi_{1}\left[\left(16 n^{2} \frac{\Lambda}{\lambda}+2 n\right) E \frac{\partial_{n} \Phi_{1}}{|x|}+\frac{d+C}{t}\right]+\frac{b \psi+K}{2 t^{2}}\left[\left(16 n^{2} \frac{\Lambda}{\lambda}-2 n\right) E \frac{\partial_{n} \Phi_{1}}{|x|}+\frac{d-C}{t}\right] \\
\geq & \left(16 n^{2} \frac{\Lambda}{\lambda}+2 n\right) E \frac{\partial_{t} \Phi_{1} \partial_{n} \Phi_{1}}{|x|}+\frac{d+C}{t} \partial_{t} \Phi_{1}+\frac{(d-C)(b \psi+K)}{2 t^{3}} .
\end{aligned}
$$

Because $\frac{\partial_{t} \Phi_{1}}{|x|} \geq \frac{f^{\prime}}{f} \partial_{n} \Phi_{1}$, and if we choose $d$ large enough, then

$$
\partial_{t} \Phi\left(H-2 \partial_{i} a^{i j} \partial_{j} \Phi\right) \geq\left(16 n^{2} \frac{\Lambda}{\lambda}+2 n\right) E \frac{f^{\prime}}{f}\left(\partial_{n} \Phi_{1}\right)^{2}+\frac{2 d}{t} \partial_{t} \Phi_{1}+\left(\frac{d}{2}-C\right) \frac{b \psi+K}{t^{3}} .
$$

And

Also

$$
\left|\partial_{t} a^{i j} \partial_{i j} \Phi\right| \leq C\left|\nabla^{2} \Phi\right| \leq C\left(\partial_{n n} \Phi_{1}+\frac{\left|\nabla^{2} \psi\right|}{t}\right) \leq C\left(\partial_{n} \Phi_{1}+\frac{1}{t}\right) .
$$

$$
-a^{i j} \partial_{i j t} \Phi=-a^{n n} \partial_{n n t} \Phi_{1}-\frac{b a^{i j}}{2 t^{2}}\left|\partial_{i j} \psi\right| \geq-a^{n n} \partial_{n n t} \Phi_{1}-\frac{C}{t^{2}}\left|\nabla^{2} \psi\right|,
$$

because $\partial_{n n t} \Phi_{1}<0,\left|\nabla^{2} \psi\right| \leq C$, then

$$
-a^{i j} \partial_{i j t} \Phi \geq-\frac{C}{t^{2}}
$$

By (53) we have

$$
\begin{aligned}
& \left|2 \partial_{i} a^{i j} \partial_{j} \Phi\left(H+a^{i j} \partial_{i j} \Phi\right)\right| \\
\leq & 2\left(n E \frac{\partial_{n} \Phi_{1}}{|x|}+\frac{C}{t}\right)\left(16 n^{2} \frac{\Lambda}{\lambda} E \frac{\partial_{n} \Phi_{1}}{|x|}+\frac{d}{t}+a^{n n} \partial_{n n} \Phi_{1}+\frac{C}{t}\left|\nabla^{2} \psi\right|\right) .
\end{aligned}
$$

Notice that $\left|\nabla^{2} \psi\right| \leq C$, and if we choose $d$ large enough, then

$$
\begin{aligned}
\left|2 \partial_{i} a^{i j} \partial_{j} \Phi\left(H+a^{i j} \partial_{i j} \Phi\right)\right| & \leq C\left(\partial_{n} \Phi_{1}+\frac{1}{t}\right)\left(\partial_{n} \Phi_{1}+\frac{d}{t}\right) \\
& \leq C\left[\left(\partial_{n} \Phi_{1}\right)^{2}+\frac{d}{t} \partial_{n} \Phi_{1}+\frac{d}{t^{2}}\right] .
\end{aligned}
$$


Using the Cauchy inequality, we obtain

$$
\left|2 \partial_{i} a^{i j} \partial_{j} \Phi\left(H+a^{i j} \partial_{i j} \Phi\right)\right| \leq C\left(\partial_{n} \Phi_{1}\right)^{2}+\frac{d^{2}}{t^{2}} .
$$

And

$$
\begin{aligned}
-\frac{1}{2} \partial_{t} H-\frac{1}{2} H^{2} & =-8 n^{2} \frac{\Lambda}{\lambda} E \frac{\partial_{n t} \Phi_{1}}{|x|}+\frac{d}{2 t^{2}}-\frac{1}{2}\left(16 n^{2} \frac{\Lambda}{\lambda} E \frac{\partial_{n} \Phi_{1}}{|x|}+\frac{d}{t}\right)^{2} \\
& \geq-8 n^{2} \frac{\Lambda}{\lambda} E \frac{\partial_{n t} \Phi_{1}}{|x|}+\frac{d}{2 t^{2}}-\left(16 n^{2} \frac{\Lambda}{\lambda} E \frac{\partial_{n} \Phi_{1}}{|x|}\right)^{2}-\frac{d^{2}}{t^{2}} \\
& \geq-8 n^{2} \frac{\Lambda}{\lambda} E \frac{\partial_{n t} \Phi_{1}}{|x|}-C\left(\partial_{n} \Phi_{1}\right)^{2}-\frac{d^{2}}{t^{2}} .
\end{aligned}
$$

Take in account that $\partial_{n t} \Phi_{1}<0$, then

$$
-\frac{1}{2} \partial_{t} H-\frac{1}{2} H^{2} \geq-C\left(\partial_{n} \Phi_{1}\right)^{2}-\frac{d^{2}}{t^{2}} .
$$

Combining them together, we obtain

$$
\begin{aligned}
J_{4} \geq & {\left[\left(16 n^{2} \frac{\Lambda}{\lambda}+2 n\right) E \frac{f^{\prime}}{f}-C\right]\left(\partial_{n} \Phi_{1}\right)^{2}+\partial_{t t} \Phi_{1}+\frac{2 d}{t} \partial_{t} \Phi_{1}-C \partial_{n} \Phi_{1} } \\
& +\frac{b \psi+K}{t^{3}}\left(\frac{d}{2}-C\right)-\frac{2 d^{2}+C}{t^{2}} \\
\geq & {\left[\left(16 n^{2} \frac{\Lambda}{\lambda}+2 n\right) E \frac{f^{\prime}}{f}-C\right]\left(\partial_{n} \Phi_{1}\right)^{2}+\partial_{t t} \Phi_{1}+\frac{2 d}{t} \partial_{t} \Phi_{1}-C \partial_{n} \Phi_{1} } \\
& +\frac{b \psi}{t^{3}}\left(\frac{d}{2}-C\right)+\frac{K d}{4 t^{3}}-\frac{2 d^{2}+C}{t^{2}} .
\end{aligned}
$$

Estimate of $J_{5}$.

$$
\begin{aligned}
J_{5}= & -2 \partial_{t}\left\langle A \nabla \Phi_{1}, \nabla \Phi_{1}\right\rangle-2 \partial_{t}\left\langle A \nabla \Phi_{2}, \nabla \Phi_{2}\right\rangle-4 \partial_{t}\left\langle A \nabla \Phi_{1}, \nabla \Phi_{2}\right\rangle \\
= & -2 \partial_{t}\left[a^{n n}\left(\partial_{n} \Phi_{1}\right)^{2}\right]-\frac{b^{2}}{2} \partial_{t}\left(\frac{a^{i j} \partial_{i} \psi \partial_{j} \psi}{t^{2}}\right)+2 b \partial_{t}\left(\frac{\partial_{n} \Phi_{1}}{t} a^{n i} \partial_{i} \psi\right) \\
= & -2 \partial_{t} a^{n n}\left(\partial_{n} \Phi_{1}\right)^{2}-4 a^{n n} \frac{f^{\prime}}{f}\left(\partial_{n} \Phi_{1}\right)^{2}-\frac{b^{2} \partial_{t} a^{i j} \partial_{i} \psi \partial_{j} \psi}{2 t^{2}}+\frac{b^{2} a^{i j} \partial_{i} \psi \partial_{j} \psi}{t^{3}} \\
& +2 b \frac{\partial_{n} \Phi_{1}}{t} \partial_{t} a^{n i} \partial_{i} \psi+2 b \partial_{t}\left(\frac{\partial_{n} \Phi_{1}}{t}\right) a^{n i} \partial_{i} \psi
\end{aligned}
$$

Because $f^{\prime}<0, a^{n n} \geq \lambda,\left|\partial_{t} a^{n n}\right| \leq M$, and

$$
\begin{gathered}
\left|\partial_{t} a^{i j} \partial_{i} \psi \partial_{j} \psi\right| \leq M \sum_{i, j}\left|\partial_{i} \psi\right|\left|\partial_{j} \psi\right| \leq M n|\nabla \psi|^{2} \\
a^{i j} \partial_{i} \psi \partial_{j} \psi \geq \lambda|\nabla \psi|^{2} \geq 0 \\
\left|\partial_{t} a^{n i} \partial_{i} \psi\right| \leq M \sum_{i}\left|\partial_{i} \psi\right| \leq M \sqrt{n}|\nabla \psi|
\end{gathered}
$$


BACKWARD UNIQUENESS FOR PARABOLIC OPERATORSWITH VARIABLE COEFFICIENTS IN A HALF SPAC25 then

$$
\begin{aligned}
J_{5} \geq & -2 M\left(\partial_{n} \Phi_{1}\right)^{2}-4 \lambda \frac{f^{\prime}}{f}\left(\partial_{n} \Phi_{1}\right)^{2}-\frac{b^{2} M n|\nabla \psi|^{2}}{2 t^{2}} \\
& -\frac{2 b}{t} M \sqrt{n} \partial_{n} \Phi_{1}|\nabla \psi|+2 \alpha b\left(\frac{f^{\prime}}{t}-\frac{f}{t^{2}}\right) \gamma x_{n}^{\alpha-1} a^{n i} \partial_{i} \psi .
\end{aligned}
$$

By the Cauchy inequality we have

$$
\frac{2 b}{t} M \sqrt{n} \partial_{n} \Phi_{1}|\nabla \psi| \leq 2 M\left(\partial_{n} \Phi_{1}\right)^{2}+\frac{b^{2} M n|\nabla \psi|^{2}}{2 t^{2}}
$$

then

$$
J_{5} \geq-4 M\left(\partial_{n} \Phi_{1}\right)^{2}-4 \lambda \frac{f^{\prime}}{f}\left(\partial_{n} \Phi_{1}\right)^{2}-\frac{b^{2} M n|\nabla \psi|^{2}}{t^{2}}+2 \alpha b\left(\frac{f^{\prime}}{t}-\frac{f}{t^{2}}\right) \gamma x_{n}^{\alpha-1} a^{n i} \partial_{i} \psi
$$

By (38), $,|\nabla \psi| \leq C|x|, a^{n i} \partial_{i} \psi \leq C x_{n}$, and notice that $f^{\prime}<0$, then we have

$$
\begin{aligned}
J_{5} & \geq-4 M\left(\partial_{n} \Phi_{1}\right)^{2}-4 \lambda \frac{f^{\prime}}{f}\left(\partial_{n} \Phi_{1}\right)^{2}-\frac{C|x|^{2}}{t^{2}}+C\left(\frac{f^{\prime}}{t}-\frac{f}{t^{2}}\right) \gamma x_{n}^{\alpha} \\
& =\left(-4 \lambda \frac{f^{\prime}}{f}-4 M\right)\left(\partial_{n} \Phi_{1}\right)^{2}-\frac{C|x|^{2}}{t^{2}}+C\left(\frac{f^{\prime}}{t f}-\frac{1}{t^{2}}\right) \Phi_{1} .
\end{aligned}
$$

Estimate of $J_{6}$.

Recall that

$$
F_{0}=2 \partial_{t} \Phi-2 a_{\epsilon}^{i j} \partial_{i j} \Phi-4 a_{\epsilon}^{i j} \partial_{i} \Phi \partial_{j} \Phi-16 n^{2} \frac{\Lambda}{\lambda} E \frac{\partial_{n} \Phi_{1}}{|x|}-\frac{d}{t} .
$$

Direct calculations show that

$$
\begin{aligned}
J_{6}= & \tilde{\Delta}\left(\partial_{t} \Phi\right)-\tilde{\Delta}\left[a_{\epsilon}^{i j}\left(\partial_{i j} \Phi+2 \partial_{i} \Phi \partial_{j} \Phi\right)\right]-8 n^{2} \frac{\Lambda}{\lambda} E \tilde{\Delta}\left(\frac{\partial_{n} \Phi_{1}}{|x|}\right) \\
= & \frac{f^{\prime}}{f} \tilde{\Delta} \Phi_{1}+\frac{b}{2 t^{2}} \tilde{\Delta} \psi-\left(a^{k l} \partial_{k l} a_{\epsilon}^{i j}+\partial_{k} a^{k l} \partial_{l} a_{\epsilon}^{i j}\right)\left(\partial_{i j} \Phi+2 \partial_{i} \Phi \partial_{j} \Phi\right) \\
& -\left(\partial_{k} a^{k l} a_{\epsilon}^{i j}+2 a^{k l} \partial_{k} a_{\epsilon}^{i j}\right)\left(\partial_{i j l} \Phi+4 \partial_{i l} \Phi \partial_{j} \Phi\right) \\
& -a^{k l} a_{\epsilon}^{i j}\left(\partial_{i j k l} \Phi+4 \partial_{i k l} \Phi \partial_{j} \Phi+4 \partial_{i k} \Phi \partial_{j l} \Phi\right) \\
& -8 n^{2} \frac{\Lambda}{\lambda} E\left[\partial_{n}^{3} \Phi_{1} \frac{a^{n n}}{|x|}+\partial_{n n} \Phi_{1}\left(\frac{\partial_{k} a^{k n}}{|x|^{2}}-\frac{2 a^{k n} x_{k}}{|x|^{3}}\right)\right. \\
& \left.+\partial_{n} \Phi_{1}\left(\frac{3 a^{k l} x_{k} x_{l}}{|x|^{5}}-\frac{\partial_{k} a^{k l} x_{l}+a^{k k}}{|x|^{3}}\right)\right] .
\end{aligned}
$$

Next we estimate the terms of $J_{6}$. By (35) and (38) we have

$$
\begin{aligned}
& \left|a_{\epsilon}^{i j}\right|,\left|\nabla a_{\epsilon}^{i j}\right| \text { and }\left|\nabla^{2} a_{\epsilon}^{i j}\right| \text { are all bounded; } \\
& \left|\nabla^{k} \psi\right| \leq \frac{C}{|x|^{k-2}}, \quad k=1,2,3,4 .
\end{aligned}
$$

Then it is easy to see

$$
\begin{gathered}
\left|\tilde{\Delta} \Phi_{1}\right|=\left|\partial_{i} a^{i n} \partial_{n} \Phi_{1}+a^{n n} \partial_{n n} \Phi_{1}\right| \leq C \partial_{n} \Phi_{1} \\
|\tilde{\Delta} \psi|=\left|\partial_{i} a^{i j} \partial_{j} \psi+a^{i j} \partial_{i j} \psi\right| \leq C\left(|\nabla \psi|+\left|\nabla^{2} \psi\right|\right) \leq C|x|
\end{gathered}
$$


and

$$
\begin{aligned}
& \left(a^{k l} \partial_{k l} a_{\epsilon}^{i j}+\partial_{k} a^{k l} \partial_{l} a_{\epsilon}^{i j}\right)\left(\partial_{i j} \Phi+2 \partial_{i} \Phi \partial_{j} \Phi\right) \\
\leq & C\left(\left|\nabla^{2} \Phi\right|+|\nabla \Phi|^{2}\right) \\
\leq & C\left[\partial_{n n} \Phi_{1}+\frac{\left|\nabla^{2} \psi\right|}{t}+\left(\partial_{n} \Phi_{1}\right)^{2}+\frac{|\nabla \psi|^{2}}{t^{2}}\right] \\
\leq & C\left[\left(\partial_{n} \Phi_{1}\right)^{2}+\partial_{n} \Phi_{1}+\frac{1}{t}+\frac{|x|^{2}}{t^{2}}\right] ;
\end{aligned}
$$

also

$$
\begin{gathered}
\left(\partial_{k} a^{k l} a_{\epsilon}^{i j}+2 a^{k l} \partial_{k} a_{\epsilon}^{i j}\right)\left(\partial_{i j l} \Phi+4 \partial_{i l} \Phi \partial_{j} \Phi\right) \\
\leq C\left(\left|\nabla^{3} \Phi\right|+|\nabla \Phi|^{2}+\left|\nabla^{2} \Phi\right|^{2}\right) \\
\leq C\left[\left|\partial_{n}^{3} \Phi_{1}\right|+\frac{\left|\nabla^{3} \psi\right|}{t}+\left(\partial_{n} \Phi_{1}\right)^{2}+\frac{|\nabla \psi|^{2}}{t^{2}}+\left(\partial_{n n} \Phi_{1}\right)^{2}+\frac{\left|\nabla^{2} \psi\right|^{2}}{t^{2}}\right] \\
\leq C\left[\left(\partial_{n} \Phi_{1}\right)^{2}+\partial_{n} \Phi_{1}+\frac{1}{t^{2}}+\frac{|x|^{2}}{t^{2}}\right] ; \\
a^{k l} a_{\epsilon}^{i j}\left(\partial_{i j k l} \Phi+4 \partial_{i k l} \Phi \partial_{j} \Phi+4 \partial_{i k} \Phi \partial_{j l} \Phi\right) \\
\leq C\left(\left|\nabla^{4} \Phi\right|+\sum_{k=1}^{3}\left|\nabla^{k} \Phi\right|^{2}\right) \\
\leq C\left[\left|\partial_{n}^{4} \Phi_{1}\right|+\frac{\left|\nabla^{4} \psi\right|}{t}+\sum_{k=1}^{3}\left(\partial_{n}^{k} \Phi_{1}\right)^{2}+\sum_{k=1}^{3} \frac{\left|\nabla^{k} \psi\right|^{2}}{t^{2}}\right] \\
\leq C\left[\left(\partial_{n} \Phi_{1}\right)^{2}+\partial_{n} \Phi_{1}+\frac{1}{t^{2}}+\frac{|x|^{2}}{t^{2}}\right] ; \\
\leq C\left(\left|\partial_{n}^{3} \Phi_{1}\right|+\partial_{n n} \Phi_{1}+\partial_{n} \Phi_{1}\right) \\
\leq C \partial_{n} \Phi_{1} .
\end{gathered}
$$

Combining them together, we have

$$
J_{6} \geq C \frac{f^{\prime}}{f} \partial_{n} \Phi_{1}-C\left[\left(\partial_{n} \Phi_{1}\right)^{2}+\partial_{n} \Phi_{1}+\frac{1}{t^{2}}+\frac{|x|^{2}}{t^{2}}\right] .
$$

Combining (52), (54), (55), (56), (57) and (다), we have

$$
\begin{aligned}
M_{2} \geq & 2\left[(\alpha-1) \lambda^{2}-\left(16 n^{2} \frac{\Lambda}{\lambda}+8 n^{2}+4 n\right) \Lambda E\right] \frac{\left(\partial_{n} \Phi_{1}\right)^{3}}{|x|} \\
& +\left[-\left(4 \lambda-\left(16 n^{2} \frac{\Lambda}{\lambda}+2 n\right) E\right) \frac{f^{\prime}}{f}-\frac{2 d \Lambda+C}{t}\right]\left(\partial_{n} \Phi_{1}\right)^{2} \\
& +\left[\partial_{t t} \Phi_{1}+\frac{2 d}{t} \partial_{t} \Phi_{1}+C \frac{f^{\prime}}{f} \partial_{n} \Phi_{1}-C \partial_{n} \Phi_{1}+C\left(\frac{f^{\prime}}{t f}-\frac{1}{t^{2}}\right) \Phi_{1}\right] \\
& +\left[\frac{b \psi}{t^{3}}\left(\frac{d}{2}-C\right)-\frac{C}{t^{3}}|x|^{2}-\frac{d b^{2} \Lambda+C}{2 t^{3}}|\nabla \psi|^{2}\right]+\frac{K d}{4 t^{3}}-\frac{2 d^{2}+C}{t^{2}} .
\end{aligned}
$$


Next we estimate the terms of the right side of the above inequality. We always choose $d$ large enough.

Notice that $\left(16 n^{2} \frac{\Lambda}{\lambda}+2 n\right) E<16 n^{2}\left(\frac{\Lambda}{\lambda}+1\right) E_{0}<\lambda$, then

$$
\begin{aligned}
& -\left(4 \lambda-\left(16 n^{2} \frac{\Lambda}{\lambda}+2 n\right) E\right) \frac{f^{\prime}}{f}-\frac{2 d \Lambda+C}{t} \\
\geq & -3 \lambda \frac{f^{\prime}}{f}-\frac{3 d \Lambda}{t} \\
= & \frac{3 \lambda K}{t\left(1-t^{K}\right)}-\frac{3 d \Lambda}{t} \\
\geq & \frac{3 \lambda K-3 d \Lambda}{t} \\
\geq & 0 .
\end{aligned}
$$

And

$$
\begin{aligned}
& \partial_{t t} \Phi_{1}+\frac{2 d}{t} \partial_{t} \Phi_{1}+C \frac{f^{\prime}}{f} \partial_{n} \Phi_{1}-C \partial_{n} \Phi_{1}+C\left(\frac{f^{\prime}}{t f}-\frac{1}{t^{2}}\right) \Phi_{1} \\
\geq & \Phi_{1}\left[\frac{f^{\prime \prime}}{f}+\frac{2 d}{t} \frac{f^{\prime}}{f}+C \frac{f^{\prime}}{f}-C+C\left(\frac{f^{\prime}}{t f}-\frac{1}{t^{2}}\right)\right] \\
\geq & \Phi_{1}\left[\frac{f^{\prime \prime}}{f}+\frac{3 d}{t} \frac{f^{\prime}}{f}-\frac{d}{t^{2}}\right] \\
= & \Phi_{1}\left[\frac{K(K+1-3 d)}{t^{2}\left(1-t^{K}\right)}-\frac{d}{t^{2}}\right] \\
\geq & \Phi_{1} \frac{K(K+1-3 d)-d}{t^{2}} \\
\geq & 0 .
\end{aligned}
$$

By (38)

$$
\psi \geq \frac{|x|^{2}}{2},|\nabla \psi| \leq 4\left(\frac{\Lambda}{\lambda}+1\right)^{2}|x|
$$

then

$$
\begin{aligned}
& \frac{b \psi}{t^{3}}\left(\frac{d}{2}-C\right)-\frac{C}{t^{3}}|x|^{2}-\frac{d b^{2} \Lambda+C}{2 t^{3}}|\nabla \psi|^{2} \\
\geq & \left(\frac{d b}{4}-C\right) \frac{|x|^{2}}{t^{3}}-C \frac{|x|^{2}}{t^{3}}-8\left(d b^{2} \Lambda+C\right)\left(\frac{\Lambda}{\lambda}+1\right)^{4} \frac{|x|^{2}}{t^{3}} \\
= & \frac{|x|^{2}}{t^{3}}\left[\frac{d b}{4}-8 d b^{2} \Lambda\left(\frac{\Lambda}{\lambda}+1\right)^{4}-C\right] \\
= & \frac{|x|^{2}}{t^{3}}\left[d b\left(\frac{1}{4}-8 b \Lambda\left(\frac{\Lambda}{\lambda}+1\right)^{4}\right)-C\right] .
\end{aligned}
$$

Since

$$
b=\frac{1}{64 \Lambda\left(\frac{\Lambda}{\lambda}+1\right)^{4}},
$$

then

$$
\frac{b \psi}{t^{3}}\left(\frac{d}{2}-C\right)-\frac{C}{t^{3}}|x|^{2}-\frac{d b^{2} \Lambda+C}{2 t^{3}}|\nabla \psi|^{2} \geq \frac{|x|^{2}}{t^{3}}\left(\frac{d b}{8}-C\right) \geq \frac{d b|x|^{2}}{16 t^{3}}
$$


Finally,

Combining them together, we have

$$
\frac{K d}{4 t^{3}}-\frac{2 d^{2}+C}{t^{2}} \geq \frac{K d}{4 t^{3}}-\frac{3 d^{2}}{t^{2}} \geq \frac{K d-12 d^{2}}{4 t^{3}} \geq \frac{1}{t^{3}} .
$$

$$
M_{2} \geq 2\left[(\alpha-1) \lambda^{2}-\left(16 n^{2} \frac{\Lambda}{\lambda}+8 n^{2}+4 n\right) \Lambda E\right] \frac{\left(\partial_{n} \Phi_{1}\right)^{3}}{|x|}+\frac{b d|x|^{2}}{16 t^{3}}+\frac{1}{t^{3}} .
$$

Estimate of $\left|\nabla\left(F-F_{0}\right)\right|$.

Recall that

$$
F-F_{0}=2\left(a_{\epsilon}^{i j}-a^{i j}\right)\left(\partial_{i j} \Phi+2 \partial_{i} \Phi \partial_{j} \Phi\right)
$$

then

$$
\begin{aligned}
\left|\nabla\left(F-F_{0}\right)\right|= & 2 \mid\left(\nabla a_{\epsilon}^{i j}-\nabla a^{i j}\right)\left(\partial_{i j} \Phi+2 \partial_{i} \Phi \partial_{j} \Phi\right) \\
& +\left(a_{\epsilon}^{i j}-a^{i j}\right)\left(\nabla \partial_{i j} \Phi+4 \partial_{i} \Phi \nabla \partial_{j} \Phi\right) \mid \\
\leq & 2\left|\nabla a_{\epsilon}^{i j}-\nabla a^{i j}\right|\left(\left|\partial_{i j} \Phi\right|+\left|\partial_{i} \Phi\right|^{2}+\left|\partial_{j} \Phi\right|^{2}\right) \\
& +2\left|a_{\epsilon}^{i j}-a^{i j}\right|\left(\left|\nabla \partial_{i j} \Phi\right|+2\left|\partial_{i} \Phi\right|^{2}+2\left|\nabla \partial_{j} \Phi\right|^{2}\right) .
\end{aligned}
$$

Since $|x| \geq 1$ in $Q$, by (35) we have

$$
\left|\nabla a_{\epsilon}^{i j}\right| \leq \frac{2 E}{|x|},\left|a_{\epsilon}^{i j}-a^{i j}\right| \leq \frac{E}{|x|},
$$

then

$$
\begin{aligned}
\left|\nabla\left(F-F_{0}\right)\right| \leq & \frac{6 E}{|x|}\left(\left|\partial_{n n} \Phi_{1}\right|+\frac{C}{t}\left|\nabla^{2} \psi\right|+2 n|\nabla \Phi|^{2}\right) \\
& +\frac{2 E}{|x|}\left(\left|\partial_{n}^{3} \Phi_{1}\right|+\frac{C}{t}\left|\nabla^{3} \psi\right|+2 n|\nabla \Phi|^{2}+2 n\left|\nabla^{2} \Phi\right|^{2}\right) \\
\leq & \frac{E}{|x|}\left[8 \partial_{n} \Phi_{1}+\frac{C}{t}\left(\left|\nabla^{2} \psi\right|+\left|\nabla^{3} \psi\right|\right)+16|\nabla \Phi|^{2}+4 n\left|\nabla^{2} \Phi\right|^{2}\right] .
\end{aligned}
$$

Next we estimate the terms of the right side of the above inequality.

By the inequality $(a+b)^{2} \leq \frac{3}{2} a^{2}+3 b^{2}$, we have

$$
\begin{gathered}
|\nabla \Phi|^{2}=\left|\nabla \Phi_{1}+\nabla \Phi_{2}\right|^{2} \leq \frac{3}{2}\left|\nabla \Phi_{1}\right|^{2}+3\left|\nabla \Phi_{2}\right|^{2} \leq \frac{3}{2}\left(\partial_{n} \Phi_{1}\right)^{2}+\frac{C|x|^{2}}{t^{2}} \\
\left|\nabla^{2} \Phi\right|^{2}=\left|\nabla^{2} \Phi_{1}+\nabla^{2} \Phi_{2}\right|^{2} \leq \frac{3}{2}\left|\nabla^{2} \Phi_{1}\right|^{2}+3\left|\nabla^{2} \Phi_{2}\right|^{2} \leq \frac{3}{2}\left(\partial_{n n} \Phi_{1}\right)^{2}+\frac{C}{t^{2}} .
\end{gathered}
$$

Then

$$
\begin{aligned}
\left|\nabla\left(F-F_{0}\right)\right| & \leq \frac{E}{|x|}\left[8 \partial_{n} \Phi_{1}+24 n\left(\partial_{n} \Phi_{1}\right)^{2}+6 n\left(\partial_{n n} \Phi_{1}\right)^{2}+\frac{C|x|^{2}}{t^{2}}\right] \\
& \leq \frac{E}{|x|}\left[8 \partial_{n} \Phi_{1}+30 n\left(\partial_{n} \Phi_{1}\right)^{2}+\frac{C|x|^{2}}{t^{2}}\right] .
\end{aligned}
$$

Because

$$
8 \partial_{n} \Phi_{1} \leq 2 n\left(\partial_{n} \Phi_{1}\right)^{2}+\frac{8}{n} \leq 2 n\left(\partial_{n} \Phi_{1}\right)^{2}+\frac{8|x|^{2}}{t^{2}}
$$


then

$$
\left|\nabla\left(F-F_{0}\right)\right| \leq \frac{E}{|x|}\left[32 n\left(\partial_{n} \Phi_{1}\right)^{2}+\frac{C|x|^{2}}{t^{2}}\right]=32 n E\left[\frac{\left(\partial_{n} \Phi_{1}\right)^{2}}{|x|}+\frac{C|x|}{t^{2}}\right] .
$$

Thus we proved Lemma 3.4.

\section{Appendix}

Appendix A: The properties of $\left\{a_{\epsilon}^{i j}\right\}$.

$$
\begin{aligned}
& a_{\epsilon}^{i j}(x, t)=\int_{\mathbb{R}^{n}} a^{i j}(x-y, t) \phi_{\epsilon}(y) d y, \text { where } \phi \text { is a mollifier and } \epsilon=\frac{1}{2} \text {, and } \\
& \qquad(x, t) \in \begin{cases}\mathbb{R}^{n} \times(0,2), & \text { under the assumptions of Proposition 1.3. } \\
\left(\mathbb{R}_{+}^{n}+e_{n}\right) \times(0,1), & \text { under the assumptions of Proposition 1.4. }\end{cases}
\end{aligned}
$$

Then $\left\{a_{\epsilon}^{i j}\right\}$ satisfy:

$$
\begin{aligned}
& \text { i) } \lambda|\xi|^{2} \leq a_{\epsilon}^{i j}(x, t) \xi_{i} \xi_{j} \leq \Lambda|\xi|^{2}, \forall \xi \in \mathbb{R}^{n} \\
& \text { ii) }\left|\nabla a_{\epsilon}^{i j}(x, t)\right| \leq M ;\left|\nabla a_{\epsilon}^{i j}(x, t)\right| \leq \frac{2 E}{|x|} \text { when }|x| \geq 1 ; \\
& \text { iii) }\left|a_{\epsilon}^{i j}(x, t)-a^{i j}(x, t)\right| \leq 2 \Lambda ;\left|a_{\epsilon}^{i j}(x, t)-a^{i j}(x, t)\right| \leq \frac{E}{|x|} \text { when }|x| \geq 1 ; \\
& \text { iv) }\left|\partial_{k l} a_{\epsilon}^{i j}(x, t)\right| \leq c(n) M ;\left|\partial_{k l} a_{\epsilon}^{i j}(x, t)\right| \leq \frac{c(n) E}{|x|} \text { when }|x| \geq 1 .
\end{aligned}
$$

Proof.

i) Obvious.

ii)

$$
\left|\nabla a_{\epsilon}^{i j}(x, t)\right| \leq \int_{\mathbb{R}^{n}}\left|\nabla a^{i j}(x-y, t)\right| \phi_{\epsilon}(y) d y \leq M \int_{\mathbb{R}^{n}} \phi_{\epsilon}(y) d y=M,
$$

and when $|x| \geq 1$,

$$
\left|\nabla a_{\epsilon}^{i j}(x, t)\right| \leq \int_{\mathbb{R}^{n}}\left|\nabla a^{i j}(x-y, t)\right| \phi_{\epsilon}(y) d y \leq \int_{\mathbb{R}^{n}} \frac{E}{|x-y|} \phi_{\epsilon}(y) d y \leq \int_{\mathbb{R}^{n}} \frac{E}{|x|-\frac{1}{2}} \phi_{\epsilon}(y) d y \leq \frac{2 E}{|x|} .
$$

iii) The first part is obvious. We only need to prove the second one.

$$
\begin{aligned}
\left|a_{\epsilon}^{i j}(x, t)-a^{i j}(x, t)\right| & \leq \int_{\mathbb{R}^{n}}\left|a^{i j}(x-y, t)-a^{i j}(x, t)\right| \phi_{\epsilon}(y) d y \\
& \leq \int_{\mathbb{R}^{n}}\left|\nabla a^{i j}(x-\theta y, t)\right||y| \phi_{\epsilon}(y) d y, \quad(0<\theta<1)
\end{aligned}
$$

and when $|x| \geq 1$,

$$
\left|a_{\epsilon}^{i j}(x, t)-a^{i j}(x, t)\right| \leq \int_{\mathbb{R}^{n}} \frac{E}{2|x-\theta y|} \phi_{\epsilon}(y) d y \leq \int_{\mathbb{R}^{n}} \frac{E}{2\left(|x|-\frac{1}{2}\right)} \phi_{\epsilon}(y) d y \leq \frac{E}{|x|} .
$$


$i v)$

$$
\begin{aligned}
\left|\partial_{k l} a_{\epsilon}^{i j}(x, t)\right| & \leq \int_{\mathbb{R}^{n}}\left|\partial_{k} a^{i j}(x-y, t) \| \partial_{l} \phi_{\epsilon}(y)\right| d y \\
& \leq \epsilon^{-n-1} \int_{\mathbb{R}^{n}}\left|\partial_{k} a^{i j}(x-y, t) \|\left(\partial_{l} \phi\right)\left(\frac{y}{\epsilon}\right)\right| d y \\
& \leq \frac{M}{\epsilon}\left\|\partial_{l} \phi\right\|_{1} \\
& \leq 2\|\nabla \phi\|_{1} M,
\end{aligned}
$$

and when $|x| \geq 1$,

$$
\begin{aligned}
\left|\partial_{k l} a_{\epsilon}^{i j}(x, t)\right| & \leq \epsilon^{-n-1} \int_{\mathbb{R}^{n}}\left|\partial_{k} a^{i j}(x-y, t)\right|\left|\left(\partial_{l} \phi\right)\left(\frac{y}{\epsilon}\right)\right| d y \\
& \leq \epsilon^{-n-1} \int_{\mathbb{R}^{n}} \frac{E}{|x-y|}\left|\left(\partial_{l} \phi\right)\left(\frac{y}{\epsilon}\right)\right| d y \\
& \leq \frac{2 E}{\epsilon|x|}\left\|\partial_{l} \phi\right\|_{1} \\
& \leq \frac{4|| \nabla \phi||_{1} E}{|x|}
\end{aligned}
$$

Appendix B: The properties of $\psi$.

Recall that $\psi(x)=|x|^{2}-2 \frac{\Lambda}{\lambda}|x| x_{n}+2\left(\frac{\Lambda}{\lambda}\right)^{2} x_{n}^{2}$. Then $\psi$ satisfies

$$
\begin{aligned}
& \text { i) } \psi \geq \frac{|x|^{2}}{2} \\
& \text { ii) } D^{2} \psi \leq C\left(\frac{\Lambda}{\lambda}\right) I_{n} ; \\
& \text { iii) }|\nabla \psi| \leq 4\left(\frac{\Lambda}{\lambda}+1\right)^{2}|x|,\left|\nabla^{k} \psi\right| \leq \frac{C\left(n, \frac{\Lambda}{\lambda}\right)}{|x|^{k-2}}, k=2,3,4 ; \\
& \text { iv) } a^{n i} \partial_{i} \psi \leq C(\Lambda, \lambda) x_{n} .
\end{aligned}
$$

Proof. Because $i$ ), the first part of $i i i$ ) and $i v$ ) play more important role in the proof of Proposition 1.4, we just prove them. The others can be proved by direct calculations. In fact

$$
\psi=\frac{|x|^{2}}{2}+\frac{1}{2}\left(|x|-2 \frac{\Lambda}{\lambda} x_{n}\right)^{2} \geq \frac{|x|^{2}}{2}
$$

and

$$
\partial_{i} \psi= \begin{cases}2\left(1-\frac{\Lambda}{\lambda} \frac{x_{n}}{|x|}\right) x_{i}, & \text { if } 1 \leq i \leq n-1 \\ -2 \frac{\Lambda}{\lambda}|x|-2 \frac{\Lambda}{\lambda} \frac{x_{n}}{|x|} x_{n}+\left[4\left(\frac{\Lambda}{\lambda}\right)^{2}+2\right] x_{n}, & \text { if } i=n\end{cases}
$$

Then

$$
\begin{aligned}
|\nabla \psi| & \leq 2\left|1-\frac{\Lambda}{\lambda} \frac{x_{n}}{|x|}\right|\left|x^{\prime}\right|+\left|-2 \frac{\Lambda}{\lambda}\right| x\left|-2 \frac{\Lambda}{\lambda} \frac{x_{n}}{|x|} x_{n}+\left[4\left(\frac{\Lambda}{\lambda}\right)^{2}+2\right] x_{n}\right| \\
& \leq 2\left(1+\frac{\Lambda}{\lambda}\right)|x|+\left[4\left(\frac{\Lambda}{\lambda}\right)^{2}+4 \frac{\Lambda}{\lambda}+2\right]|x| \\
& \leq 4\left(\frac{\Lambda}{\lambda}+1\right)^{2}|x| .
\end{aligned}
$$


Direct calculations show that

$$
a^{n i} \partial_{i} \psi=2 \sum_{i=1}^{n-1} a^{n i} x_{i}-2 \frac{\Lambda}{\lambda} a^{n n}|x|-2 \frac{\Lambda}{\lambda} \frac{x_{n}}{|x|} \sum_{i=1}^{n} a^{n i} x_{i}+\left[4\left(\frac{\Lambda}{\lambda}\right)^{2}+2\right] a^{n n} x_{n} .
$$

By the Cauchy inequality we have

$$
\left|\sum_{i=1}^{n} a^{n i} x_{i}\right| \leq \sqrt{\sum_{i=1}^{n}\left(a^{n i}\right)^{2} \sum_{i=1}^{n} x_{i}^{2}} \leq \Lambda|x|
$$

Similarly, we have

$$
\left|\sum_{i=1}^{n-1} a^{n i} x_{i}\right| \leq \Lambda\left|x^{\prime}\right|
$$

Since

$$
\lambda \leq a^{n n} \leq \Lambda
$$

then

$$
\begin{aligned}
a^{n i} \partial_{i} \psi & \leq 2 \Lambda\left|x^{\prime}\right|-2 \Lambda|x|+2 \frac{\Lambda^{2}}{\lambda} x_{n}+\left[4\left(\frac{\Lambda}{\lambda}\right)^{2}+2\right] \Lambda x_{n} \\
& \leq\left[4\left(\frac{\Lambda}{\lambda}\right)^{2}+2 \frac{\Lambda}{\lambda}+2\right] \Lambda x_{n}
\end{aligned}
$$

\section{REFERENCES}

[1] N. Aronszajn, A. Krzywicki and J. Szarski, A unique continuation theorem for exterior differential forms on Riemannian manifolds, Ark. Mat. 4(1962), 417-453.

[2] B. F. Jones, A fundamental solution of the heat equation which is supported in a strip, J. Math. Anal. Appl. 60 (1977), 314C324.

[3] W. Littman, Boundary control theory for hyperbolic and parabolic partial differential equations with constant coefficients, Annali Scuola Norm. Sup. Pisa Serie IV, 3 (1978), 567C580.

[4] L. Hörmander, Uniqueness theorems for second order elliptic differential equations, Comm. Partial Differential Equations 8(1983), 21-64.

[5] X. Y. Chen, A strong unique continuation theorem for parabolic equations, Math. Ann. 311(1996), 603-630.

[6] C. C. Poon, Unique continuation for parabolic equations, Comm. Partial Differential Equations 21(1996), 521-539.

[7] L. Escauriaza, G. A. Seregin and V. Šverák, Backward uniqueness for parabolic equations, Arch. Ration. Mech. Anal., 169(2003), no. 2, 147-157.

[8] L. Escauriaza, G. A. Seregin and V. Šverák, Backward uniqueness for the heat operator in a half-space, Algebra i Analiz 15 (2003), no. 1, 201-214; translation in St. Petersburg Math. J. 15(2004), no. 1, 139-148.

[9] L. Escauriaza, G. A. Seregin and V. Šverák, $L^{3, \infty}$ solutions to the Navier-Stokes equations and backward uniqueness, Russ. Math. Surv., 58(2003), no. 2, 211-250.

[10] L. Escauriaza and F. J. Fernández, Unique continuation for parabolic operators, Ark. Mat., 41(2003), no. 1, 35-60.

[11] L. Escauriaza, C. E. Kenig, G. Ponce and L. Vega, Decay at infinity of caloric functions within characteristic hyperplanes, Math. Res. Lett. 13(2006), no. 3, 441-453.

[12] L. Escauriaza, Carleman inequalities and the heat operator, Duke Math. J. 104(2000), 113-127.

[13] L. Escauriaza and L. Vega, Carleman inequalities and the heat operator II, Indiana Univ. Math. J. 50(2001), 1149-1169.

[14] J. C. Saut and B. Scheurer, Unique continuation for evolution equations, J. Diff. Equ., 66(1987), 118-137. 
[15] C. D. Sogge, A unique continuation theorem for second order parabolic differential operators, Ark. Mat. 28(1990), 159-182.

[16] $\mathrm{Lu} \mathrm{Li}$ and V. Šverák, Backward uniqueness for the heat equation in cones, Comm. Partial Differential Equations, 37 (2012), no. 8, 1414-1429.

[17] E. M. Landis and O. A. Oleinik, Generalized analyticity and some related properties of solutions of elliptic and parabolic equations, Russian Math. Surveys 29(1974), 195-212.

[18] Tu A. Nguyen, On a question of Landis and Oleinik, Tran. Amer. Math. Soc., 362(2010), no. 6, 2875-2899.

[19] F. H. Lin, A uniqueness theorem for parabolic equations, Comm. Pure Appl. Math. 42(1988), $125-136$.

[20] A. Plis, On non-uniqueness in Cauchy problems for an elliptic second order differential equation, Bull. Acad. Polon. Sci. Math. Astronom. Phys. 11(1963), 95-100.

[21] K. Miller, Non-unique continuation for certain ode's in Hilbert space and for uniformly parabolic and elliptic equations in self-adjoint divergence form, Arch. Rational Mech. Anal. 54(1963), 105117.

[22] N. Mandache, On a counterexample concerning unique continuation for elliptic equations in divergence form, Mathematical Physics, Analysis and Geometry. 1(1998), $273 \mathrm{C} 292$.

[23] L. C. Evans, Partial Differential Equations, Amer. Math. Soc., 1998.

[24] O. A. Ladyženskaja and V. A. Solonnikov, Linear and quasilinear equations of parabolic type, Translations of mathematical monographs, Amer. Math. Soc., 1968.

\section{Jie $\mathrm{Wu}$}

Institute of Mathematics, Academy of Mathematics and Systems Science, Chinese Academy of Sciences, Beijing 100190, P. R. China.

Email address: jackwu@amss.ac.cn

\section{Liqun Zhang}

Hua Loo-Keng Key Laboratory of Mathematics, Chinese Academy of Sciences, Beijing 100190, P. R. China.

Email address: lqzhang@amss.ac.cn 